\title{
Intermolecular Radical Addition and Addition/Cyclization Reactions of Alkoxyamines onto Nonactivated Alkenes
}

\author{
Christian Wetter, Katja Jantos, Katharina Woithe and Armido Studer* \\ Department of Chemistry, Philipps-University Marburg, 35032 Marburg,
}

\section{Supporting Information}

General. ${ }^{1} \mathrm{H}$ - and ${ }^{13} \mathrm{C}-\mathrm{NMR}$ spectra: recorded on a Bruker ARX 500, ARX 400, ARX 300, ARX 200 or a AC 200. Chemical shifts $\delta$ in ppm rel. to $\mathrm{SiMe}_{4}$ as internal standard. TLC: Merck silica gel $60 \mathrm{~F}_{254}$ plates; detection with UV or dipping into a soln. of $\mathrm{KMnO}_{4}(1.5 \mathrm{~g}$ in $333 \mathrm{~mL} 1 \mathrm{M} \mathrm{NaOH})$ or a soln. of $\mathrm{Ce}\left(\mathrm{SO}_{4}\right)_{2} \cdot \mathrm{H}_{2} \mathrm{O}(10 \mathrm{~g})$, phosphormolybdic acid hydrate $(25 \mathrm{~g})$, conc. $\mathrm{H}_{2} \mathrm{SO}_{4}(60 \mathrm{~mL})$, and $\mathrm{H}_{2} \mathrm{O}(940 \mathrm{~mL})$, followed by heating. FC: Merck or Fluka silica gel $60(40-63 \mu \mathrm{m})$; at ca. 0.4 bar. GC: Hewlett Packard 5890 chromatograph using Hewlett Packard HP-5, Macherey-Nagel Optima $\delta-\square 3$, or Supelco $\gamma$-DEX 120 columns. Melting points: Büchi 510 apparatus; uncorrected. I.R. spectra: recorded on a Perkin Elmer 782 or Bruker IFS-200 spectrophotometer. MS: Recorded on a VG Tribid, Varian CH7 (EI); IonSpec Ultima, Finnigan MAT TSQ 700 or a Finnigan MAT 95S (ESI) in m/z (\% of basis peak). Solvents were purified by standard methods. Compounds sensitive to air and moisture were handled under argon using Schlenk techniques.

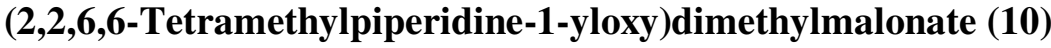

A soln. of diisopropylamine $(2.16 \mathrm{~mL}, 15.36 \mathrm{mmol})$ in 1,2-dimethoxyethane $(60 \mathrm{~mL})$ was treated with $\mathrm{BuLi}(15.36 \mathrm{mmol})$ at $-60{ }^{\circ} \mathrm{C}$; a white precipitate was formed. After stirring for 30 min. at $-60{ }^{\circ} \mathrm{C}$, dimethyl malonate $(1.6 \mathrm{~mL}, 13.96 \mathrm{mmol})$ was added dropwise. The precipitate disappeared. After stirring for another $30 \mathrm{~min} .$, 2,2,6,6-tetramethylpiperidin-1-oxyl radical (TEMPO) (2.40 g, $15.36 \mathrm{mmol})$ and subsequently anhydrous $\mathrm{CuCl}_{2}(2.06 \mathrm{~g}, 15.36$ mmol) were added. The reaction mixture turned brown. After stirring for $3 \mathrm{~h}$ at $0{ }^{\circ} \mathrm{C}$, the reaction mixture was treated with a sat. $\mathrm{NH}_{4} \mathrm{Cl}$ soln. and extracted with diethyl ether. The organic layer was washed with brine and dried $\left(\mathrm{MgSO}_{4}\right)$. Evaporation of the solvents in vacuo and purification of the crude product by FC ( $t$-butylmethylether/pentane $=1: 4$ ) afforded the 
alkoxyamine 10 (3.53 g, 88\%) as colorless crystals. M.p. $81-82{ }^{\circ}$ C. I.R. (film): $2963 s, 1769 s$, 1745s, 1262s, 1093s, 1020s, 797s. ${ }^{1} \mathrm{H}-\mathrm{NMR}\left(200 \mathrm{MHz}, \mathrm{CDCl}_{3}\right): 4.90\left(s, 2 \mathrm{H}, \mathrm{CH}_{2}\right) ; 3.72(s$, $\left.6 \mathrm{H}, \mathrm{CH}_{3}\right) ; 1.59-1.0\left(m, b r .6 \mathrm{H}, \mathrm{CH}_{2}\right) ; 1.20\left(s, b r .6 \mathrm{H}, \mathrm{CH}_{3}\right) ; 0.99\left(s, b r .6 \mathrm{H}, \mathrm{CH}_{3}\right) .{ }^{13} \mathrm{C}-$ NMR (75 MHz, $\mathrm{CDCl}_{3}$ ): 167.6, 86.4, 60.3, 52.5, 40.1, 32.5, 20.1, 16.9; MS (ESI): 326 (70 $\left.[\mathrm{M}+\mathrm{K}]^{+}\right), 310\left(100[\mathrm{M}+\mathrm{Na}]^{+}\right), 288\left(45[\mathrm{M}+\mathrm{H}]^{+}\right)$. HRMS (ESI) calcd for $\mathrm{C}_{14} \mathrm{H}_{25} \mathrm{NNaO}_{5}$ $\left([\mathrm{M}+\mathrm{Na}]^{+}\right): 310.1630$. Found: 310.1629 .

\section{(2,2,6,6-Tetramethylpiperidine-1-yloxy)di-tert-butylmalonate (11)}

Diisopropylamine $(2.07 \mathrm{~mL}, 14.88 \mathrm{mmol})$ in 1,2-dimethoxyethane was deprotonated with $\operatorname{BuLi}\left(14.88 \mathrm{mmol}, 1.7 \mathrm{M}\right.$ in hexanes) at $0{ }^{\circ} \mathrm{C}$; a white precipitate was formed. After stirring for $30 \mathrm{~min}$. at $0{ }^{\circ} \mathrm{C}$, di-tert-butyl malonate $(3.0 \mathrm{~mL}, 13.39 \mathrm{mmol})$ was added dropwise. The precipitate disappeared. After stirring for another $30 \mathrm{~min}$., TEMPO $(2.33 \mathrm{~g}, 14.88 \mathrm{mmol})$ and subsequently anhydrous $\mathrm{CuCl}_{2}(2.00 \mathrm{~g}, 14.88 \mathrm{mmol})$ were added. The reaction mixture turned brown. After stirring for $1 \mathrm{~h}$ at $0{ }^{\circ} \mathrm{C}$ and $18 \mathrm{~h}$ at $\mathrm{rt}$, the reaction mixture was treated with a sat. $\mathrm{NH}_{4} \mathrm{Cl}$ soln. and was extracted with diethyl ether. The organic layer was washed with brine and dried $\left(\mathrm{MgSO}_{4}\right)$. Evaporation of the solvents in vacuo and purification of the crude product by FC (ether/pentane $=1: 20)$ afforded the alkoxyamine $11(4.20 \mathrm{~g}, 84 \%)$ as colorless crystals. M.p. $97-99{ }^{\circ}$ C. I.R. (film): $2980 s, 2938 s, 1758 s, 1734 s, 1473 m, 1371 m, 1230 m, 1132 \mathrm{~m} \mathrm{~cm}^{-1}$. ${ }^{1} \mathrm{H}-\mathrm{NMR}\left(200 \mathrm{MHz}, \mathrm{CDCl}_{3}\right): 4.69(s, 1 \mathrm{H}, \mathrm{CH}) ; 1.63-1.23\left(m, 6 \mathrm{H}, \mathrm{CH}_{2}\right) ; 1.46(m, 18 \mathrm{H}$, $\left.\mathrm{CH}_{3}\right) ; 1.19\left(s, 6 \mathrm{H}, \mathrm{CH}_{3}\right) ; 1.09\left(s, 6 \mathrm{H}, \mathrm{CH}_{3}\right) .{ }^{13} \mathrm{C}-\mathrm{NMR}\left(75 \mathrm{MHz}, \mathrm{CDCl}_{3}\right): 166.6(\mathrm{C}), 87.6$ $(\mathrm{CH}), 82.1(\mathrm{C}), 60.2(\mathrm{C}), 40.1\left(\mathrm{CH}_{2}\right), 32.6\left(\mathrm{CH}_{3}\right), 27.9\left(\mathrm{CH}_{3}\right), 20.1\left(\mathrm{CH}_{3}\right), 17.0\left(\mathrm{CH}_{2}\right) . \mathrm{MS}$ (ESI): $410\left(13,[\mathrm{M}+\mathrm{K}]^{+}\right), 394\left(100,[\mathrm{M}+\mathrm{Na}]^{+}\right), 372\left(42,[\mathrm{M}+\mathrm{H}]^{+}\right), 316$ (18), 260 (55). HRMS (ESI) calcd for $\mathrm{C}_{20} \mathrm{H}_{37} \mathrm{NNaO}_{5}\left([\mathrm{M}+\mathrm{Na}]^{+}\right)$: 394.2569. Found: 394.2566.

\section{Addition products 12, 14, 15, 17, 19, 21, 34 and 38: General Procedure 1 (GP 1)}

Alkoxyamine 10 and the alkene (5 equiv.) were dissolved in 1,2-dichloroethane (DCE) (1 M solution) under an argon atmosphere. The soln. was heated to $135^{\circ} \mathrm{C}$ in a sealed tube $(5 \mathrm{~mL})$ for $3 \mathrm{~d}$. The reaction mixture was then allowed to cool to $\mathrm{rt}$ and diethyl ether was added. Removal of the solvents and purification by FC afforded the product.

\section{Addition products 13, 16, 18, 20, 35 and 39: General Procedure 2 (GP 2)}

Alkoxyamine 11 and the alkene (5 equiv.) were dissolved in tert-butanol $(t-\mathrm{BuOH})(1 \mathrm{M}$ solution) under an argon atmosphere. The soln. was heated to $135^{\circ} \mathrm{C}$ in a sealed tube $(5 \mathrm{~mL})$ for $5 \mathrm{~d}$. The reaction mixture was then allowed to cool to $\mathrm{rt}$ and diethyl ether was added. 
Removal of the solvents and purification by FC afforded the product.

\section{Cyclization Products 26 - 33: General Procedure (GP 3)}

A solution of the alkoxyamine (10 or 11) and the diene (22-25) in $t$-BuOH $(0.08 \mathrm{~mL})$ or DCE $(0.09 \mathrm{~mL})$ was heated for $48 \mathrm{~h}$ at $135^{\circ} \mathrm{C}$ (sealed tube). Removal of the solvent and purification by flash-chromatography (FC) afforded the desired product.

\section{2-[2-(2,2,6,6-Tetramethylpiperidine-1-yloxy)octyl]dimethylmalonate (12)}

According to GP 1, with 10 (100 mg, $0.348 \mathrm{mmol})$ and 1-octene (195 mg, $1.74 \mathrm{mmol})$ in DCE $(0.35 \mathrm{~mL}) . \mathrm{FC}$ (ether/pentane = 1:20) afforded 12 (91 mg, 66\%). I.R. (film): 3462w, 2929s, $2871 m, 1756 s, 1739 s, 1436 m, 1360 m, 1241 m, 1154 w \mathrm{~cm}^{-1} .{ }^{1} \mathrm{H}-\mathrm{NMR}$ (200 MHz, $\mathrm{CDCl}_{3}$ ): 3.78-3.71 (m, $2 \mathrm{H}, 2 \mathrm{x} \mathrm{CH}) ; 3.73\left(s, 3 \mathrm{H}, \mathrm{CH}_{3}\right) ; 3.72\left(s, 3 \mathrm{H}, \mathrm{CH}_{3}\right) ; 2.17-2.09\left(m, 2 \mathrm{H}, \mathrm{CH}_{2}\right)$; 2.07-2.03 (m, $\left.1 \mathrm{H}, \mathrm{CH}_{2}\right) ; 1.50-1.42(m, 5 \mathrm{H}) ; 1.41-1.26(m, 10 \mathrm{H}) ; 1.08-1.04(m, 12 \mathrm{H})$; 0.90-0.84 (m, $3 \mathrm{H}) .{ }^{13} \mathrm{C}-\mathrm{NMR}\left(50 \mathrm{MHz}, \mathrm{CDCl}_{3}\right)$ : $170.0(\mathrm{C}), 169.9(\mathrm{C}), 78.9(\mathrm{CH}), 59.9(\mathrm{C})$, $59.0(\mathrm{C}), 52.3\left(\mathrm{CH}_{3}\right), 52.2\left(\mathrm{CH}_{3}\right), 48.5(\mathrm{CH}), 40.2\left(\mathrm{CH}_{2}\right), 40.0\left(\mathrm{CH}_{2}\right), 34.0\left(2 \mathrm{x} \mathrm{CH}_{3}\right), 32.9$ $\left(\mathrm{CH}_{2}\right), 32.6\left(\mathrm{CH}_{2}\right), 31.7\left(\mathrm{CH}_{2}\right), 29.3\left(\mathrm{CH}_{2}\right), 25.7\left(\mathrm{CH}_{2}\right), 22.4\left(\mathrm{CH}_{2}\right), 20.4\left(\mathrm{CH}_{3}\right), 20.2\left(\mathrm{CH}_{3}\right)$, $17.1\left(\mathrm{CH}_{2}\right), 13.9\left(\mathrm{CH}_{3}\right)$. MS (ESI): $438\left(5,[\mathrm{M}+\mathrm{K}]^{+}\right), 422\left(21,[\mathrm{M}+\mathrm{Na}]^{+}\right), 400\left(100,[\mathrm{M}+\mathrm{H}]^{+}\right)$, 276 (11), 158 (8), 126 (42). HRMS (ESI) calcd for $\mathrm{C}_{22} \mathrm{H}_{42} \mathrm{NO}_{5}\left([\mathrm{M}+\mathrm{H}]^{+}\right)$: 400.3063. Found: 400.3058 .

\section{2-[2-(2,2,6,6-Tetramethylpiperidin-1-yloxy)octyl]tert-butylmethylmalonate}

According to GP 1, with $10(100 \mathrm{mg}, 0.348 \mathrm{mmol})$ and 1 -octene $(195 \mathrm{mg}, 1.74 \mathrm{mmol})$ in $t$ $\mathrm{BuOH}(0.35 \mathrm{~mL})$. FC (ether/pentane $=1: 25)$ afforded the transesterification product $(55 \mathrm{mg}$, 36\%) besides 12 (49 mg, 35\%). I.R. (film): 3447w, 2930s, 2871m, 1751s, 1734s, 1457m, $1369 m, 1257 m, 1146 s \mathrm{~cm}^{-1} .{ }^{1} \mathrm{H}-\mathrm{NMR}\left(200 \mathrm{MHz}, \mathrm{CDCl}_{3}\right): 3.80-3.60$ (m, $\left.2 \mathrm{H}, 2 \mathrm{x} \mathrm{CH}\right) ; 3.72(s$, $3 \mathrm{H}, \mathrm{CH}_{3}$ isomer $\left.\mathrm{A}\right) ; 3.71\left(s, 3 \mathrm{H}, \mathrm{CH}_{3}\right.$ isomer $\left.\mathrm{B}\right) ; 2.13-1.96\left(m, 3 \mathrm{H}, \mathrm{CH}_{2}\right) ; 1.52-1.38(m, 5$ $\left.\mathrm{H}, \mathrm{CH}_{2}\right) ; 1.47\left(s, 9 \mathrm{H}, \mathrm{CH}_{3}\right) ; 1.35-1.17\left(m, 10 \mathrm{H}, \mathrm{CH}_{2}\right) ; 1.13-1.02\left(m, 12 \mathrm{H}, \mathrm{CH}_{3}\right) ; 0.90-0.84$ $(m, 3 \mathrm{H}) .{ }^{13} \mathrm{C}-\mathrm{NMR}\left(50 \mathrm{MHz}, \mathrm{CDCl}_{3}\right): 170.8$ (C, isomer A), $170.6(\mathrm{C}$, isomer $\mathrm{B}), 169.0(\mathrm{C}$, isomer A), $168.7(\mathrm{C}$, isomer $\mathrm{B}), 81.7(\mathrm{C}$, isomer $\mathrm{A}), 81.6(\mathrm{C}$, isomer $\mathrm{B}), 79.0(\mathrm{CH}$, isomer $\mathrm{A})$, $78.9\left(\mathrm{CH}\right.$, isomer B), $60.1(\mathrm{C}), 59.2(\mathrm{C}), 52.2(\mathrm{CH}$ isomer $\mathrm{A}), 52.1(\mathrm{CH}$ isomer $\mathrm{B}), 50.0\left(\mathrm{CH}_{3}\right.$ isomer A), $49.7\left(\mathrm{CH}_{3}\right.$ isomer B), $40.4\left(\mathrm{CH}_{2}\right), 40.2\left(\mathrm{CH}_{2}\right), 34.2\left(\mathrm{CH}_{3}\right), 34.1\left(\mathrm{CH}_{3}\right), 33.2\left(\mathrm{CH}_{2}\right.$ isomer A), $32.9\left(\mathrm{CH}_{2}\right.$ isomer $\left.\mathrm{B}\right), 32.8\left(\mathrm{CH}_{2}\right.$ isomer $\left.\mathrm{A}\right), 32.7\left(\mathrm{CH}_{2}\right.$ isomer $\left.\mathrm{B}\right), 31.9\left(\mathrm{CH}_{2}\right), 29.6$ $\left(\mathrm{CH}_{2}\right), 27.9\left(\mathrm{CH}_{3}\right), 26.0\left(\mathrm{CH}_{2}\right.$ isomer A), $25.8\left(\mathrm{CH}_{2}\right.$ isomer $\left.\mathrm{B}\right), 20.4\left(\mathrm{CH}_{3}\right), 20.2\left(\mathrm{CH}_{3}\right), 17.3$ $\left(\mathrm{CH}_{2}\right), 14.1\left(\mathrm{CH}_{3}\right) . \mathrm{MS}(\mathrm{ESI}): 480\left(3,[\mathrm{M}+\mathrm{K}]^{+}\right), 464\left(13,[\mathrm{M}+\mathrm{Na}]^{+}\right), 442\left(100,[\mathrm{M}+\mathrm{H}]^{+}\right), 386$ 
(87), 342 (10), 126 (25). HRMS (ESI) calcd for $\mathrm{C}_{25} \mathrm{H}_{48} \mathrm{NO}_{5}\left([\mathrm{M}+\mathrm{H}]^{+}\right)$: 442.3533. Found: 442.3532 .

\section{2-[2-(2,2,6,6-Tetramethylpiperidin-1-yloxy)octyl]di-tert-butylmalonate (13)}

According to GP 2, with $11(100 \mathrm{mg}, 0.27 \mathrm{mmol})$ and 1-octene $(151 \mathrm{mg}, 1.35 \mathrm{mmol})$ in $t$ $\mathrm{BuOH}(0.27 \mathrm{~mL})$. FC (ether/pentane = 1:50) afforded $13(94 \mathrm{mg}, 72 \%)$ besides unreacted starting material (19 mg, 19\%). I.R. (film): 3411w, 2976s, 2930s, 1729s, 1457w, 1368m, $1256 m, 1136 \mathrm{~cm}^{-1} .{ }^{1} \mathrm{H}-\mathrm{NMR}\left(200 \mathrm{MHz}, \mathrm{CDCl}_{3}\right): 3.79-3.67(m, 1 \mathrm{H}, \mathrm{CH}) ; 3.54(t, J=7.5$ $\mathrm{Hz}, 1 \mathrm{H}, \mathrm{CH}) ; 2.02\left(d \times d, J_{1}=8.3 \mathrm{~Hz}, J_{2}=6.5 \mathrm{~Hz}, 2 \mathrm{H}, \mathrm{CH}_{2}\right) ; 1.57-1.38(m, 6 \mathrm{H}) ; 1.46(s, 18$ $\left.\mathrm{H}, \mathrm{C}\left(\mathrm{CH}_{3}\right)\right) ; 1.37-1.17(m, 10 \mathrm{H}) ; 1.16-1.04(m, 12 \mathrm{H}) ; 0.91-0.84(m, 3 \mathrm{H}) .{ }^{13} \mathrm{C}-\mathrm{NMR}(50$ $\mathrm{MHz}, \mathrm{CDCl}_{3}$ ): $169.4(\mathrm{C}), 169.1(\mathrm{C}), 81.3(\mathrm{C}), 81.0(\mathrm{C}), 78.8(\mathrm{CH}), 60.2(\mathrm{C}), 59.3$ (C), 51.0 $(\mathrm{CH}), 40.4\left(\mathrm{CH}_{2}\right), 40.2\left(\mathrm{CH}_{2}\right), 34.2\left(\mathrm{CH}_{3}\right), 34.1\left(\mathrm{CH}_{3}\right), 33.0\left(\mathrm{CH}_{2}\right), 32.9\left(\mathrm{CH}_{2}\right), 31.9\left(\mathrm{CH}_{2}\right)$, $29.6\left(\mathrm{CH}_{2}\right), 27.9\left(\mathrm{CH}_{3}\right), 25.9\left(\mathrm{CH}_{2}\right), 22.7\left(\mathrm{CH}_{2}\right), 20.6\left(2 \mathrm{x} \mathrm{CH}_{3}\right), 17.4\left(\mathrm{CH}_{2}\right), 14.1\left(\mathrm{CH}_{3}\right) . \mathrm{MS}$ (ESI): $506\left(7,[\mathrm{M}+\mathrm{Na}]^{+}\right), 484\left(100,[\mathrm{M}+\mathrm{H}]^{+}\right), 428$ (22), 372 (12), 195 (5). HRMS (ESI) calcd for $\mathrm{C}_{28} \mathrm{H}_{54} \mathrm{NO}_{5}\left([\mathrm{M}+\mathrm{H}]^{+}\right)$: 484.4002. Found: 400.4002 .

\section{2-[4-Phenyl-2-(2,2,6,6-tetramethylpiperidin-1-yloxy)butyl]dimethylmalonate (14)}

According to GP 1, with 10 (100 mg, $0.348 \mathrm{mmol})$ and 4-phenyl-1-butene (230 mg, 1.74 mmol) in DCE $(0.35 \mathrm{~mL})$. FC (ether/pentane $=1: 20$ to 1:10 ) afforded $14(71 \mathrm{mg}, 49 \%)$. I.R. (film): $3465 w, 3062 w, 2932 m, 1738 s, 1618 w, 1496 w, 1436 s, 1360 m, 1243 s, 1153 s \mathrm{~cm}^{-1} .{ }^{1} \mathrm{H}-$ NMR (200 MHz, $\left.\mathrm{CDCl}_{3}\right)$ : 7.28-7.13 (m, $\left.5 \mathrm{H}, \mathrm{CH}\right) ; 3.78-3.70(m, 2 \mathrm{H}, \mathrm{CH}) ; 3.74(s, 3 \mathrm{H}$, $\left.\mathrm{CH}_{3}\right) ; 3.73\left(s, 3 \mathrm{H}, \mathrm{CH}_{3}\right) ; 2.70-2.34\left(m, 2 \mathrm{H}, \mathrm{CH}_{2}\right) ; 2.23\left(d \times d, J_{1}=7.2 \mathrm{~Hz}, J_{2}=5.5 \mathrm{~Hz}, 2 \mathrm{H}\right.$, $\left.\mathrm{CH}_{2}\right) ; 1.65-1.25\left(m, 8 \mathrm{H}, \mathrm{CH}_{2}\right) ; 1.10\left(s, 3 \mathrm{H}, \mathrm{CH}_{3}\right) ; 1.04\left(s, 3 \mathrm{H}, \mathrm{CH}_{3}\right) ; 1.03\left(s, 3 \mathrm{H}, \mathrm{CH}_{3}\right)$; $0.98\left(s, 3 \mathrm{H}, \mathrm{CH}_{3}\right) .{ }^{13} \mathrm{C}-\mathrm{NMR}\left(50 \mathrm{MHz}, \mathrm{CDCl}_{3}\right): 170.1(\mathrm{C}), 170.0(\mathrm{C}), 142.1(\mathrm{CH}), 128.3$ $(\mathrm{CH}), 128.2(\mathrm{CH}), 125.6(\mathrm{CH}), 78.3(\mathrm{CH}), 60.1(\mathrm{C}), 59.3(\mathrm{C}), 52.5\left(\mathrm{CH}_{3}\right), 52.4\left(\mathrm{CH}_{3}\right), 48.6$ $(\mathrm{CH}), 40.3\left(\mathrm{CH}_{2}\right), 40.1\left(\mathrm{CH}_{2}\right), 34.3\left(\mathrm{CH}_{2}\right), 33.9\left(2 x \mathrm{CH}_{3}\right), 33.0\left(\mathrm{CH}_{2}\right), 32.1\left(\mathrm{CH}_{2}\right) 20.5\left(\mathrm{CH}_{3}\right)$, $20.4\left(\mathrm{CH}_{3}\right), 17.3\left(\mathrm{CH}_{2}\right)$. MS (ESI): $458\left(11,[\mathrm{M}+\mathrm{Ka}]^{+}\right), 442\left(88,[\mathrm{M}+\mathrm{Na}]^{+}\right), 420\left(83,[\mathrm{M}+\mathrm{H}]^{+}\right)$, 285 (62). HRMS (ESI) calcd for $\mathrm{C}_{24} \mathrm{H}_{38} \mathrm{NO}_{5}\left([\mathrm{M}+\mathrm{H}]^{+}\right)$: 420.2750 . Found: 420.2743.

\section{2-[2-Butoxy-2-(2,2,6,6-tetramethylpiperidin-1-yloxy)ethyl]dimethylmalonate (15)}

According to GP 1, with 10 (100 mg, $0.348 \mathrm{mmol})$, butyl vinyl ether (174 mg, $1.74 \mathrm{mmol})$ and $\mathrm{KOH}(2 \mathrm{mg})$ in DCE $(0.35 \mathrm{~mL})$. FC (ether/pentane = 1:20) afforded $15(105 \mathrm{mg}, 78 \%)$. I.R. (film): $3471 w, 2933 s, 2872 m, 1740 s, 1463 m, 1362 m, 1260 s, 1242 m, 1155 w \mathrm{~cm}^{-1} .{ }^{1} \mathrm{H}-$ $\operatorname{NMR}\left(500 \mathrm{MHz}, \mathrm{CDCl}_{3}\right): 4.79\left(d \times d, J_{l}=6.0 \mathrm{~Hz}, J_{2}=3.9 \mathrm{~Hz}, 1 \mathrm{H}, \mathrm{CH}\right) ; 3.83\left(d \times t, J_{l}=9.2\right.$ 
$\left.\mathrm{Hz}, J_{2}=6.6 \mathrm{~Hz}, 1 \mathrm{H}, \mathrm{OCH}_{2}\right) ; 3.72\left(s, 3 \mathrm{H}, \mathrm{CO}_{2} \mathrm{CH}_{3}\right) ; 3.71\left(s, 3 \mathrm{H}, \mathrm{CO}_{2} \mathrm{CH}_{3}\right) ; 3.65\left(d \times d, J_{1}=\right.$ $\left.6.4 \mathrm{~Hz}, J_{2}=3.6 \mathrm{~Hz}, 1 \mathrm{H}, \mathrm{CH}\right) ; 3.41\left(d \times t, J_{1}=9.2 \mathrm{~Hz}, J_{2}=6.9 \mathrm{~Hz}, 1 \mathrm{H}, \mathrm{CH}_{2}\right) ; 2.29-2.18(m, 2$ $\left.\mathrm{H}, \mathrm{CH}_{2}\right) ; 1.60-1.27(m, 10 \mathrm{H}) ; 1.18(s, 3 \mathrm{H}) ; 1.06(t, J=17.6 \mathrm{~Hz}, 9 \mathrm{H}) ; 0.89(t, J=7.55 \mathrm{~Hz}, 3$ $\left.\mathrm{H}, \mathrm{CH}_{3}\right) .{ }^{13} \mathrm{C}-\mathrm{NMR}\left(125 \mathrm{MHz}, \mathrm{CDCl}_{3}\right): 170.0(\mathrm{C}), 169.9(\mathrm{C}), 104.7(\mathrm{CH}), 70.3\left(\mathrm{CH}_{2}\right), 60.6$ (C), $59.3(\mathrm{C}), 52.4\left(\mathrm{CH}_{3}\right), 52.3\left(\mathrm{CH}_{3}\right), 47.1(\mathrm{CH}), 40.4\left(\mathrm{CH}_{2}\right), 40.1\left(\mathrm{CH}_{2}\right), 33.6\left(\mathrm{CH}_{3}\right), 33.2$ $\left(\mathrm{CH}_{3}\right), 32.8\left(\mathrm{CH}_{2}\right), 31.9\left(\mathrm{CH}_{2}\right), 20.4\left(\mathrm{CH}_{3}\right), 20.0\left(\mathrm{CH}_{3}\right), 19.2\left(\mathrm{CH}_{2}\right), 17.2\left(\mathrm{CH}_{2}\right), 13.9\left(\mathrm{CH}_{3}\right)$. MS (ESI): $410\left(100,[\mathrm{M}+\mathrm{Na}]^{+}\right), 388\left(92,[\mathrm{M}+\mathrm{H}]^{+}\right), 158$ (97). HRMS (ESI) calcd for $\mathrm{C}_{20} \mathrm{H}_{38} \mathrm{NO}_{6}\left([\mathrm{M}+\mathrm{H}]^{+}\right): 388.2699$. Found: 388.2702.

\section{2-[2-Butoxy-2-(2,2,6,6-tetramethylpiperidin-1-yloxy)ethyl]di-tert-butylmalonate (16)}

According to GP 2, with 11 (100 mg, $0.27 \mathrm{mmol})$, butyl vinyl ether (134 mg, $1.35 \mathrm{mmol})$ and $\mathrm{KOH}(2 \mathrm{mg})$ in $t$-BuOH $(0.27 \mathrm{~mL})$. FC (ether/pentane $=1: 50)$ afforded 16 (88 mg, 69\%). I.R. (film): $3475 w, 2977 s, 2933 s, 2873 m, 1745 m, 1729 m, 1457 w, 1368 m, 1138 w \mathrm{~cm}^{-1} .{ }^{1} \mathrm{H}-\mathrm{NMR}$ $\left(200 \mathrm{MHz}, \mathrm{CDCl}_{3}\right): 4.78\left(d \times d, J_{1}=7.2 \mathrm{~Hz}, J_{2}=4.0 \mathrm{~Hz}, 1 \mathrm{H}, \mathrm{CH}\right) ; 3.90\left(d \times t, J_{1}=9.2 \mathrm{~Hz}, J_{2}=\right.$ $\left.6.8 \mathrm{~Hz}, 1 \mathrm{H}, \mathrm{OCH}_{2}\right) ; 3.48\left(d \times t, J_{1}=9.3 \mathrm{~Hz}, J_{2}=6.8 \mathrm{~Hz}, 1 \mathrm{H}, \mathrm{OCH}_{2}\right) ; 3.38\left(d \times d, J_{1}=9.3 \mathrm{~Hz}\right.$, $\left.J_{2}=5.5 \mathrm{~Hz}, 1 \mathrm{H}, \mathrm{CH}\right) ; 2.26-1.98\left(m, 2 \mathrm{H}, \mathrm{CH}_{2}\right) ; 1.60-1.25(m, 10 \mathrm{H}) ; 1.45(s, 18 \mathrm{H}$, $\left.\mathrm{C}\left(\mathrm{CH}_{3}\right)_{3}\right) ; 1.21(s, 3 \mathrm{H}) ; 1.09(t, J=6.8 \mathrm{~Hz}, 9 \mathrm{H}) ; 0.91\left(t, J=7.0 \mathrm{~Hz}, 3 \mathrm{H}, \mathrm{CH}_{3}\right) .{ }^{13} \mathrm{C}-\mathrm{NMR}(50$ $\left.\mathrm{MHz}, \mathrm{CDCl}_{3}\right)$ : 168.3 (2x C), $105.0(\mathrm{CH}), 81.3(\mathrm{C}), 81.2(\mathrm{C}), 70.7\left(\mathrm{CH}_{2}\right), 60.5(\mathrm{C}), 59.3(\mathrm{C})$, $50.0(\mathrm{CH}), 40.4\left(\mathrm{CH}_{2}\right), 40.2\left(\mathrm{CH}_{2}\right), 33.9\left(\mathrm{CH}_{3}\right), 33.2\left(\mathrm{CH}_{3}\right), 32.8\left(\mathrm{CH}_{2}\right), 31.9\left(\mathrm{CH}_{2}\right), 27.9$ $\left(\mathrm{CH}_{3}\right), 27.8\left(\mathrm{CH}_{3}\right), 20.5\left(\mathrm{CH}_{3}\right), 20.0\left(\mathrm{CH}_{3}\right), 19.2\left(\mathrm{CH}_{2}\right), 17.3\left(\mathrm{CH}_{2}\right), 14.0\left(\mathrm{CH}_{3}\right)$. MS (ESI): $494\left(17,[\mathrm{M}+\mathrm{Na}]^{+}\right), 472\left(100,[\mathrm{M}+\mathrm{H}]^{+}\right), 394(3), 227$ (7), 158 (11). HRMS (ESI) calcd for $\mathrm{C}_{26} \mathrm{H}_{50} \mathrm{NO}_{6}\left([\mathrm{M}+\mathrm{H}]^{+}\right): 472.3638$. Found: 472.3625 .

\section{2-[5-Methoxycarbonyloxy-2-(2,2,6,6-tetramethylpiperidin-1-yloxy)pentyl]dimethyl- malonate (17)}

According to GP 1, with $\mathbf{1 0}(100 \mathrm{mg}, 0.348 \mathrm{mmol})$ and methyl-4-pentenylcarbonate $(250 \mathrm{mg}$, $1.74 \mathrm{mmol})$ in DCE $(0.35 \mathrm{~mL})$. FC (ether/pentane = 1:3) afforded $17(63 \mathrm{mg}, 42 \%)$. I.R. (film): $3449 w, 2977 s, 2933 s, 1749 s, 1444 m, 1369 m, 1268 s, 1139 s \mathrm{~cm}^{-1} .{ }^{1} \mathrm{H}-\mathrm{NMR}(200 \mathrm{MHz}$, $\left.\mathrm{CDCl}_{3}\right): 4.13\left(d \times t, J_{1}=6.8 \mathrm{~Hz}, J_{2}=2.0 \mathrm{~Hz}, 2 \mathrm{H}, \mathrm{CH}_{2}\right) ; 3.80-3.66(m, 2 \mathrm{H}, \mathrm{CH}) ; 3.76(s, 3 \mathrm{H}$, $\left.\mathrm{CO}_{2} \mathrm{CH}_{3}\right) ; 3.73\left(s, 3 \mathrm{H}, \mathrm{CO}_{2} \mathrm{CH}_{3}\right) ; 3.72\left(s, 3 \mathrm{H}, \mathrm{CO}_{2} \mathrm{CH}_{3}\right) ; 2.27-1.99\left(m, 3 \mathrm{H}, \mathrm{CH}_{2}\right) ; 1.74-1.59$ $\left(m, 3 \mathrm{H}, \mathrm{CH}_{2}\right)$; 1.49-1.25 ( $\left.m, 6 \mathrm{H}, \mathrm{CH}_{2}\right) ; 1.07-1.02\left(m, 12 \mathrm{H}, \mathrm{CH}_{3}\right) .{ }^{13} \mathrm{C}-\mathrm{NMR}(50 \mathrm{MHz}$, $\left.\mathrm{CDCl}_{3}\right)$ : $170.0(\mathrm{C}), 169.9(\mathrm{C}), 155.8(\mathrm{C}), 78.5(\mathrm{CH}), 68.1\left(\mathrm{CH}_{2}\right), 60.6(\mathrm{C}), 59.3(\mathrm{C}), 54.6$ $\left(\mathrm{CH}_{3}\right), 52.4\left(\mathrm{CH}_{3}\right), 52.3\left(\mathrm{CH}_{3}\right), 48.6(\mathrm{CH}), 40.3\left(\mathrm{CH}_{2}\right), 40.2\left(\mathrm{CH}_{2}\right), 34.1\left(2 \mathrm{x} \mathrm{CH}_{3}\right), 32.9\left(\mathrm{CH}_{2}\right)$, $29.0\left(\mathrm{CH}_{2}\right), 25.2\left(\mathrm{CH}_{2}\right), 20.6\left(\mathrm{CH}_{3}\right), 20.4\left(\mathrm{CH}_{3}\right), 17.3\left(\mathrm{CH}_{2}\right)$. MS (ESI): $454\left(15,[\mathrm{M}+\mathrm{Na}]^{+}\right)$, 
$432\left(23,[\mathrm{M}+\mathrm{H}]^{+}\right), 126(100)$. HRMS (ESI) calcd for $\mathrm{C}_{21} \mathrm{H}_{38} \mathrm{NO}_{8}\left([\mathrm{M}+\mathrm{H}]^{+}\right):$432.2597. Found: 432.2589 .

\section{2-[5-Methoxycarbonyloxy-2-(2,2,6,6-tetramethylpiperidin-1-yloxy)pentyl]di-tert-}

\section{butylmalonate (18)}

According to GP 2, with 11 (100 mg, $0.27 \mathrm{mmol})$ and methyl-4-pentenylcarbonate (195 mg, $1.35 \mathrm{mmol})$ in $t$-BuOH $(0.27 \mathrm{~mL})$. FC (ether/pentane $=1: 12)$ afforded 18 (53 mg, 38\%). I.R. (film): 3449w, 2977s, 2933s, 1749s, 1444m, 1367m, 1268s, 1139s cm ${ }^{-1} .{ }^{1} \mathrm{H}-\mathrm{NMR}$ (400 MHz, $\left.\mathrm{CDCl}_{3}\right)$ : 4.18-4.11 (m, $\left.2 \mathrm{H}, \mathrm{CH}_{2}\right) ; 3.80-3.77(m, 1 \mathrm{H}, \mathrm{CH}) ; 3.76\left(s, 3 \mathrm{H}, \mathrm{CH}_{3}\right) ; 3.50\left(d \times d, J_{l}=\right.$ $\left.8.9 \mathrm{~Hz}, J_{2}=5.8 \mathrm{~Hz}, 1 \mathrm{H}, \mathrm{CH}\right) ; 2.14-1.93\left(m, 3 \mathrm{H}, \mathrm{CH}_{2}\right) ; 1.76-1.63\left(m, 3 \mathrm{H}, \mathrm{CH}_{2}\right) ; 1.60-1.24$ $\left(m, 6 \mathrm{H}, \mathrm{CH}_{2}\right) ; 1.45$ (s, $\left.18 \mathrm{H}, \mathrm{CH}_{3}\right), 1.10-1.06\left(m, 12 \mathrm{H}, \mathrm{CH}_{3}\right) .{ }^{13} \mathrm{C}-\mathrm{NMR}\left(100 \mathrm{MHz}, \mathrm{CDCl}_{3}\right)$ : $169.1(\mathrm{C}), 168.9(\mathrm{C}), 155.8(\mathrm{C}), 81.4(\mathrm{C}), 81.2(\mathrm{C}), 78.3(\mathrm{CH}), 68.6\left(\mathrm{CH}_{2}\right), 60.0(\mathrm{C}), 59.4(\mathrm{C})$, $54.6\left(\mathrm{CH}_{3}\right), 50.8(\mathrm{CH}), 40.3\left(\mathrm{CH}_{2}\right), 40.2\left(\mathrm{CH}_{2}\right), 34.2\left(\mathrm{CH}_{3}\right), 34.1\left(\mathrm{CH}_{3}\right), 32.7\left(\mathrm{CH}_{2}\right), 29.1$ $\left(\mathrm{CH}_{2}\right), 27.9\left(\mathrm{CH}_{3}\right), 25.2\left(\mathrm{CH}_{2}\right), 20.6(2 \mathrm{x} \mathrm{CH}), 17.3\left(\mathrm{CH}_{2}\right)$. MS (ESI): $\left.538(13 \text {, [M+Na] }]^{+}\right), 516$ $\left(65,[\mathrm{M}+\mathrm{H}]^{+}\right), 460$ (47), $404(89), 126(100)$. HRMS (ESI) calcd for $\mathrm{C}_{27} \mathrm{H}_{50} \mathrm{NO}_{8}\left([\mathrm{M}+\mathrm{H}]^{+}\right)$: 516.3536. Found: 516.3515.

\section{2-[5-(tert-Butyldimethylsilanyloxy)-2-(2,2,6,6-tetramethylpiperidin-1-} yloxy)pentyl]dimethylmalonate (19)

According to GP 1, with $\mathbf{1 0}(100 \mathrm{mg}, 0.348 \mathrm{mmol})$ and tert-butyldimethylpent-4enyloxysilane (349 mg, $1.74 \mathrm{mmol})$ in DCE $(0.35 \mathrm{~mL})$. FC (ether/pentane =1:15) afforded 19 (119 mg, 70\%). I.R. (film): 3471w, 2953s, 2931s, 2857m, 1757s, 1740s, 1436w, 1361w, $1255 m, 1099 m 836 \mathrm{~cm}^{-1} .{ }^{1} \mathrm{H}-\mathrm{NMR}\left(200 \mathrm{MHz}, \mathrm{CDCl}_{3}\right): 3.82-3.70(m, 2 \mathrm{H}, 2 \mathrm{x} \mathrm{CH}) ; 3.75(s$, $\left.3 \mathrm{H}, \mathrm{CO}_{2} \mathrm{CH}_{3}\right) ; 3.74\left(s, 3 \mathrm{H}, \mathrm{CO}_{2} \mathrm{CH}_{3}\right) ; 3.61\left(t, J=5.1 \mathrm{~Hz}, 2 \mathrm{H}, \mathrm{CH}_{2} \mathrm{OSi}\right) ; 1.56-1.17(m, 10$ $\mathrm{H}) ; 1.09-1.04(m, 12 \mathrm{H}) ; 0.89\left(s, 9 \mathrm{H}, \mathrm{C}\left(\mathrm{CH}_{3}\right)_{3}\right) ; 0.04\left(s, 6 \mathrm{H}, \mathrm{Si}\left(\mathrm{CH}_{3}\right)_{2}\right) .{ }^{13} \mathrm{C}-\mathrm{NMR}(50 \mathrm{MHz}$, $\left.\mathrm{CDCl}_{3}\right): 169.9(\mathrm{C}), 169.8(\mathrm{C}), 78.8(\mathrm{CH}), 62.9\left(\mathrm{CH}_{2}\right), 59.9(\mathrm{C}), 59.0(\mathrm{C}), 52.2\left(\mathrm{CH}_{3}\right), 52.1$ $\left(\mathrm{CH}_{3}\right), 48.4(\mathrm{CH}), 40.1\left(\mathrm{CH}_{2}\right), 39.9\left(\mathrm{CH}_{2}\right), 33.8\left(2 \times \mathrm{CH}_{3}\right), 32.9\left(\mathrm{CH}_{2}\right), 29.0(\mathrm{C}), 28.8\left(\mathrm{CH}_{2}\right)$, $25.7\left(\mathrm{CH}_{3}\right), 20.3\left(\mathrm{CH}_{3}\right), 20.2\left(\mathrm{CH}_{3}\right), 18.0\left(\mathrm{CH}_{2}\right), 17.1\left(\mathrm{CH}_{2}\right),-5.5(2 \mathrm{x} \mathrm{CH}) . \mathrm{MS}(\mathrm{ESI}): 523$ (7, $\left.[\mathrm{M}+\mathrm{K}]^{+}\right), 510\left(42,[\mathrm{M}+\mathrm{Na}]^{+}\right), 488\left(100,[\mathrm{M}+\mathrm{H}]^{+}\right), 364(26), 353$ (63), $158(85)$. HRMS (ESI) calcd for $\mathrm{C}_{25} \mathrm{H}_{50} \mathrm{NO}_{6} \mathrm{Si}\left([\mathrm{M}+\mathrm{H}]^{+}\right)$: 488.3407. Found: 488.3381 . 


\section{2-[5-(tert-Butyldimethylsilanyloxy)-2-(2,2,6,6-tetramethylpiperidin-1-yloxy)pentyl]di-}

\section{tert-butylmalonate (20)}

According to GP 2, with 11 (100 mg, $0.27 \mathrm{mmol})$ and tert-butyldimethylpent-4-enyloxysilane $(270 \mathrm{mg}, 1.35 \mathrm{mmol})$ in $t$-BuOH $(0.27 \mathrm{~mL})$. FC (ether/pentane = 1:20) afforded $20(85 \mathrm{mg}$, 55\%). I.R. (film): 3447w, 2931s, 2858s, 2143w, 1729s, 1473m, 1368m, 1255s, $1138 \mathrm{~s} \mathrm{~cm}^{-1}$. ${ }^{1} \mathrm{H}-\mathrm{NMR}\left(200 \mathrm{MHz}, \mathrm{CDCl}_{3}\right): 3.78-3.73(m, 1 \mathrm{H}, \mathrm{CH}) ; 3.65-3.57\left(m, 2 \mathrm{H}, \mathrm{CH}_{2}\right) ; 3.54\left(d \times d, J_{1}\right.$ $\left.=8.7 \mathrm{~Hz}, J_{2}=6.4 \mathrm{~Hz}, 1 \mathrm{H}, \mathrm{CH}\right) ; 2.05-2.01\left(m, 2 \mathrm{H}, \mathrm{CH}_{2}\right) ; 1.59-1.41\left(m, 8 \mathrm{H}, \mathrm{CH}_{2}\right) ; 1.45(s, 9$ $\left.\mathrm{H}, \mathrm{CH}_{3}\right) ; 1.44\left(s, 9 \mathrm{H}, \mathrm{CH}_{3}\right) ; 1.33-1.19\left(m, 2 \mathrm{H}, \mathrm{CH}_{2}\right) ; 1.09\left(s, 6 \mathrm{H}, \mathrm{CH}_{3}\right) ; 1.06\left(s, 6 \mathrm{H}, \mathrm{CH}_{3}\right)$; $0.90\left(s, 9 \mathrm{H}, \mathrm{CH}_{3}\right) ; 0.04\left(s, 6 \mathrm{H}, \mathrm{CH}_{3}\right) .{ }^{13} \mathrm{C}-\mathrm{NMR}\left(50 \mathrm{MHz}, \mathrm{CDCl}_{3}\right): 169.3(\mathrm{C}), 169.0(\mathrm{C})$, $81.2(\mathrm{C}), 81.0(\mathrm{C}), 78.8(\mathrm{CH}), 63.2\left(\mathrm{CH}_{2}\right), 60.0(\mathrm{C}), 59.2(\mathrm{C}), 50.9(\mathrm{CH}), 40.2\left(2 \mathrm{x} \mathrm{CH}_{2}\right), 34.1$ (2x CH $\left.\mathrm{CH}_{3}\right), 32.9\left(\mathrm{CH}_{2}\right), 29.3(\mathrm{C}), 29.1\left(\mathrm{CH}_{2}\right), 27.9\left(\mathrm{CH}_{3}\right), 26.0\left(\mathrm{CH}_{3}\right), 20.6\left(2 \mathrm{x} \mathrm{CH}_{3}\right), 18.3$ $\left(\mathrm{CH}_{2}\right), 17.3\left(\mathrm{CH}_{2}\right),-5.3(2 \mathrm{x} \mathrm{CH})$. MS (ESI): $572\left(100,[\mathrm{M}+\mathrm{H}]^{+}\right), 516$ (77), 460 (84), 126 (67). HRMS (ESI) calcd for $\mathrm{C}_{31} \mathrm{H}_{62} \mathrm{NO}_{6} \mathrm{Si}\left([\mathrm{M}+\mathrm{H}]^{+}\right)$: 572.4346 . Found: 572.4318 .

\section{2-[2-(2,2,6,6-Tetramethylpiperidine-1-yloxy)cyclohexyl]dimethylmalonate (21)}

According to GP 1, with 10 (100 mg, $0.348 \mathrm{mmol})$ and cyclohexene (143 mg, $1.74 \mathrm{mmol})$ in DCE $(0.35 \mathrm{~mL})$. FC (ether/pentane = 1:20) afforded trans-21 (50 mg, 39\%) and cis-21 (24 mg, 19\%). trans-21: I.R. (film): 3466w, 2932s, 2870m, 1737s, 1452m, 1435m, 1360m, $1241 m, 1137 w \mathrm{~cm}^{-1} .{ }^{1} \mathrm{H}-\mathrm{NMR}\left(400 \mathrm{MHz}, \mathrm{CDCl}_{3}\right): 3.89\left(d \times t, J_{l}=10.9 \mathrm{~Hz}, J_{2}=4.1 \mathrm{~Hz}, 1 \mathrm{H}\right.$, $\mathrm{CH}) ; 3.81(d, J=10.5 \mathrm{~Hz}, 1 \mathrm{H}, \mathrm{CH}) ; 3.73\left(s, 3 \mathrm{H}, \mathrm{CH}_{3}\right) ; 3.72\left(s, 3 \mathrm{H}, \mathrm{CH}_{3}\right) ; 2.98\left(d \times d, J_{1}=\right.$ $\left.10.5 \mathrm{~Hz}, J_{2}=4.1 \mathrm{~Hz}, 1 \mathrm{H}, \mathrm{CH}_{2}\right) ; 2.11\left(d \times d, J_{l}=13.0 \mathrm{~Hz}, J_{2}=3.8 \mathrm{~Hz}, 1 \mathrm{H}, \mathrm{CH}_{2}\right) ; 1.78-1.75$ (m, $\left.1 \mathrm{H}, \mathrm{CH}_{2}\right) ; 1.64-0.91$ (m, $\left.24 \mathrm{H}\right) .{ }^{13} \mathrm{C}-\mathrm{NMR}$ (100 MHz, $\left.\mathrm{CDCl}_{3}\right): 169.7$ (C), 169.0 (C), 82.4 $(\mathrm{CH}), 60.2(\mathrm{C}), 58.5(\mathrm{C}), 52.4\left(\mathrm{CH}_{3}\right), 52.3\left(\mathrm{CH}_{3}\right), 51.2(\mathrm{CH}), 40.5\left(\mathrm{CH}_{2}\right), 40.2\left(\mathrm{CH}_{2}\right), 38.6$ $(\mathrm{CH}), 34.9\left(\mathrm{CH}_{3}\right), 34.2\left(\mathrm{CH}_{3}\right), 28.7\left(\mathrm{CH}_{2}\right), 27.5\left(\mathrm{CH}_{2}\right), 24.6\left(\mathrm{CH}_{2}\right), 21.2\left(\mathrm{CH}_{2}\right), 20.5\left(\mathrm{CH}_{3}\right)$, $20.3\left(\mathrm{CH}_{3}\right), 17.3\left(\mathrm{CH}_{2}\right)$. MS (ESI): $408\left(61,[\mathrm{M}+\mathrm{K}]^{+}\right), 392\left(100,[\mathrm{M}+\mathrm{Na}]^{+}\right), 370\left(27,[\mathrm{M}+\mathrm{H}]^{+}\right)$, 167 (17), 142 (18), 126 (51). HRMS (ESI) calcd for $\mathrm{C}_{20} \mathrm{H}_{36} \mathrm{NO}_{5}\left([\mathrm{M}+\mathrm{H}]^{+}\right): 370.2593$. Found: 370.2612. cis-21: I.R. (film): 3466w, 2932s, 2869m, 1738s, 1683m, 1434m, 1376m, 1235m, $1134 w \mathrm{~cm}^{-1} .{ }^{1} \mathrm{H}-\mathrm{NMR}\left(400 \mathrm{MHz}, \mathrm{CDCl}_{3}\right): 4.03(d, J=5.1 \mathrm{~Hz}, 1 \mathrm{H}, \mathrm{CH}) ; 3.73\left(s, 3 \mathrm{H}, \mathrm{CH}_{3}\right)$; $3.72\left(s, 3 \mathrm{H}, \mathrm{CH}_{3}\right) ; 3.73-3.67(m, 1 \mathrm{H}, \mathrm{CH}) ; 2.64-2.43(m, 1 \mathrm{H}, \mathrm{CH}) ; 2.20-2.12(m, 1 \mathrm{H}$, $\left.\mathrm{CH}_{2}\right) ; 1.74-1.61(m, 3 \mathrm{H}), 1.52-1.33(m, 6 \mathrm{H}), 1.31-1.13(m, 4 \mathrm{H}), 1.12-1.01(m, 12 \mathrm{H}) .{ }^{13} \mathrm{C}-$ NMR (100 MHz, $\left.\mathrm{CDCl}_{3}\right)$ : $170.1(\mathrm{C}), 169.4(\mathrm{C}), 81.4(\mathrm{CH}), 60.6(\mathrm{C}), 58.6(\mathrm{C}), 52.1\left(\mathrm{CH}_{3}\right)$, $52.0\left(\mathrm{CH}_{3}\right), 51.8(\mathrm{CH}), 43.7(\mathrm{CH}) 40.7\left(\mathrm{CH}_{2}\right), 40.1\left(\mathrm{CH}_{2}\right), 34.3\left(\mathrm{CH}_{3}\right), 33.9\left(\mathrm{CH}_{3}\right), 31.3$ $\left(\mathrm{CH}_{2}\right), 27.5\left(\mathrm{CH}_{2}\right), 25.9\left(\mathrm{CH}_{2}\right), 24.6\left(\mathrm{CH}_{2}\right), 20.7\left(\mathrm{CH}_{3}\right), 20.2\left(\mathrm{CH}_{3}\right), 17.3\left(\mathrm{CH}_{2}\right) . \mathrm{MS}(\mathrm{ESI})$ : $408\left(39,[\mathrm{M}+\mathrm{K}]^{+}\right), 392\left(67,[\mathrm{M}+\mathrm{Na}]^{+}\right), 370\left(100,[\mathrm{M}+\mathrm{H}]^{+}\right), 167$ (17), 142 (18), 126 (83). 
HRMS (ESI) calcd for $\mathrm{C}_{20} \mathrm{H}_{36} \mathrm{NO}_{5}\left([\mathrm{M}+\mathrm{H}]^{+}\right)$: 370.2593. Found: 370.2580 .

\section{3-(2,2-Bismethoxycarbonyl-ethyl)-4-(2,2,6,6-tetramethylpiperidin-1-yloxymethyl)-} cyclopentane-1,1-dicarboxylic acid dimethyl ester (26)

According to GP 3 with $10(100 \mathrm{mg}, 0.35 \mathrm{mmol})$ and $22(221 \mathrm{mg}, 1.04 \mathrm{mmol})$ in DCE $(0.09 \mathrm{~mL})$ and purification by $\mathrm{FC}\left(\mathrm{SiO}_{2}\right.$, pentane $/ t$-butylmethylether $\left.=4: 1\right)$ afforded 26 (107 mg, 68\%) as cis:trans mixture of diastereoisomers. I.R. (film): 3469w, 2954s, 1735s, $1643 w, 1436 s, 1260 \mathrm{sm}^{-1} .{ }^{1} \mathrm{H}-\mathrm{NMR}\left(300 \mathrm{MHz} \mathrm{CDCl}_{3}\right)$ : both diastereomeres: $3.76-3.35$ ( $\mathrm{m}$, $1 \mathrm{H}) ; 3.67 ; 3.66 ; 3.65 ; 3.64\left(s, 12 \mathrm{H}, \mathrm{CH}_{3} \mathrm{O}\right) ; 3.53\left(d d, J_{1}=7.3 \mathrm{~Hz}, J_{2}=9.3,1 \mathrm{H}, \mathrm{H}_{2} \mathrm{CON}\right)$; $3.43\left(d d, J_{l}=7.0 \mathrm{~Hz}, J_{2}=8.3,1 \mathrm{H}, \mathrm{H}_{2} \mathrm{CON}\right) ; 2.49-1.67(m, 8 \mathrm{H}) ; 1.51-0.99(m, 18 \mathrm{H}$, TEMPO). ${ }^{13} \mathrm{C}-\mathrm{NMR}\left(75 \mathrm{MHz}, \mathrm{CDCl}_{3}\right.$ ): both diastereomeres: 173.0, 172.7, 169.8, 169.6, 59.8, 52.7, 52.5, 50.5, 44.8, 40.8, 39.7, 39.0, 38.5, 33.2, 33.0, 29.2, 20.1, 17.1; EI-MS: 484 (4) [M+Me], 343 (12) [M+-TEMPO], 311 (29), 278 (100), 139 (80). EI-HRMS: calcd for $\mathrm{C}_{24} \mathrm{H}_{38} \mathrm{NO}_{9}$ $\left(\left[\mathrm{M}^{+}-\mathrm{Me}\right]\right): 484.2547$. Found: 484.2551.

\section{3-(2,2-Bis-tert-butoxycarbonyl-ethyl)-4-(2,2,6,6-tetramethyl-piperidin-1-yloxymethyl) cyclopentane-1,1-dicarboxylic acid dimethyl ester (27)}

According to GP 3 with $11(100 \mathrm{mg}, 0.27 \mathrm{mmol})$ and $22(171 \mathrm{mg}, 0.81 \mathrm{mmol})$ in $t$-BuOH and purification by $\mathrm{FC}\left(\mathrm{SiO}_{2}\right.$, pentane/t-butylmethylether $=20: 1, t$-butylmethylether $)$ afforded 27 (63 mg, $40 \%$ ) as a 2.2:1 cis:trans mixture of diastereoisomers and the corresponding telomer 27 ( $\mathrm{n}=2$ ) (22 mg, 10\%). I. R. (film): 2976s, 2933s, 1735s, 1456w, 1436w, 1393w, 1369m, $1256 s, 1164 s, 1138 s, 1036 w, 994 w, 956 w, 849 w \mathrm{~cm}^{-1} .{ }^{1} \mathrm{H}-\mathrm{NMR}\left(300 \mathrm{MHz}, \mathrm{CDCl}_{3}\right)$ : both diastereoisomers: 3.68-3.18 (m, $3 \mathrm{H}) ; 3.61\left(s, 12 \mathrm{H}, \mathrm{CH}_{3} \mathrm{O}\right) ; 2.44-0.78(m, 14 \mathrm{H}) ; 1.35(s, 18$ $\left.\mathrm{H}, \mathrm{C}\left(\mathrm{CH}_{3}\right)_{3}\right) ; 1.04$ (s, 6H, $\mathrm{CH}_{3}$ (TEMPO)); 0.98 ( $s, 6 \mathrm{H}, \mathrm{CH}_{3}$ (TEMPO)). ${ }^{13} \mathrm{C}-\mathrm{NMR}(75 \mathrm{MHz}$, $\mathrm{CDCl}_{3}$ ): both diastereoisomers: 173.0, 172.9, 172.8, 168.91, 168.86, 168.6, 81.3, 81.2, 76.6, 59.82, 59.77, 52.8, 45.1, 40.6, 40.2, 40.0, 39.8, 38.9, 38.8, 37.7, 37.1, 33.2, 33.1, 29.0, 28.1, 27.9, 20.2, 17.1. MS (ESI): $584\left(100,[\mathrm{M}+\mathrm{H}]^{+}\right)$, HRMS (ESI): calcd for $\mathrm{C}_{31} \mathrm{H}_{54} \mathrm{NO}_{9}\left([\mathrm{M}+\mathrm{H}]^{+}\right)$: 584.3799. Found: 584.3783; corresponding telomer $30(\mathrm{n}=2)$ : MS (ESI): $833\left(14,[\mathrm{M}+\mathrm{K}]^{+}\right)$, $818\left(17,[\mathrm{M}+\mathrm{Na}]^{+}\right), 796\left(100,[\mathrm{M}+\mathrm{H}]^{+}\right)$. HRMS (ESI): calcd for $\mathrm{C}_{42} \mathrm{H}_{70} \mathrm{NO}_{13}\left([\mathrm{M}+\mathrm{H}]^{+}\right)$: 796.4847. Found: 796.4820. 


\section{2-[4-(2,2,6,6-Tetramethyl-piperidin-1-yloxymethyl)-tetrahydro-furan-3-ylmethyl]}

dimethylmalonate (28)

According to GP 3 with 10 (154 mg, $0.54 \mathrm{mmol})$ and 23 (636 mg, $3.23 \mathrm{mmol})$ in DCE (0.18 $\mathrm{mL})$ and purification by $\mathrm{FC}\left(\mathrm{SiO}_{2}\right.$, pentane/t-butylmethylether $=7: 1$, pentane $/ t$ butylmethylether $=4: 1)$ afforded $28(69 \mathrm{mg}, 33 \%)$ as a 1.8:1 cis:trans mixture of diastereoisomers. I.R. (film): 3472w, 2932s, 1738s, 1437m, 1244m, 1154m, 1048w, 771m cm ${ }^{1}$. ${ }^{1} \mathrm{H}-\mathrm{NMR}\left(300 \mathrm{MHz}, \mathrm{CDCl}_{3}\right)$ : both diastereoisomers: $3.96-3.56\left(m, 5 \mathrm{H}, \mathrm{CH}_{2} \mathrm{ON}\right.$, $\left.\mathrm{CH}\left(\mathrm{CO}_{2} \mathrm{Me}\right)_{2}, \mathrm{CH}_{2} \mathrm{O}\right) ; 3.73\left(s, 6 \mathrm{H}, \mathrm{OCH}_{3}\right) ; 3.45$ - $3.36\left(m, 2 \mathrm{H}, \mathrm{CH}_{2} \mathrm{O}\right) ; 2.54-1.86$ (br $m, 4$ $\left.\mathrm{H}, \mathrm{CH}, \mathrm{CH}_{2}\right) ; 1.62-1.08$ ( $\left.m, 18 \mathrm{H}, \mathrm{TEMPO}\right) .{ }^{13} \mathrm{C}-\mathrm{NMR}\left(75 \mathrm{MHz}, \mathrm{CDCl}_{3}\right)$ : both diastereoisomers: 169.9, 169.8, 75.3, 73.5, 72.0, 71.6, 71.2, 60.2, 52.9, 51.1, 50.9, 45.2, 41.3, 40.9, 40.1, 40.0, 39.6, 33.5, 32.9, 28.3, 27.4, 20.4, 17.4. MS (ESI): 408 (100, [M+Na] $\left.{ }^{+}\right), 386$ $\left(35,[\mathrm{M}+\mathrm{H}]^{+}\right), 126(75)$. HRMS (ESI): calcd for $\mathrm{C}_{20} \mathrm{H}_{35} \mathrm{NNaO}_{6}\left([\mathrm{M}+\mathrm{Na}]^{+}\right)$: 408.2362. Found: 408.2365 .

\section{2-[4-(2,2,6,6-Tetrametyl-piperidin-1-yloxymethyl)-tetrahydro-furan-3-ylmethyl]di-tert-} butylmalonate (29)

According to GP 3 with 11 (100 mg, $0.27 \mathrm{mmol})$ and 23 (159 mg, $0.81 \mathrm{mmol})$ in $t$-BuOH and purification by $\mathrm{FC}\left(\mathrm{SiO}_{2}\right.$, pentane/t-butylmethylether $=15: 1$, pentane/t-butylmethylether $=$ 5:1, t-butylmethylether) afforded $29(47 \mathrm{mg}, 37 \%)$ as a 1.7:1 cis:trans mixture of diastereoisomers and the corresponding telomer 29 ( $\mathrm{n}=2)$ (18 mg, 12\%). I.R. (film): 2977s, $2932 s, 2872 s, 1729 s, 1471 w, 1456 w, 1392 w, 1369 m, 1256 m, 1141 s, 1058 w, 994 w, 957 w$, 925w, 847w. ${ }^{1} \mathrm{H}-\mathrm{NMR}\left(200 \mathrm{MHz}, \mathrm{CDCl}_{3}\right)$ : both diastereomeres: 3.90-3.08 (m, $\left.7 \mathrm{H}\right)$; 2.44$0.88(m, 10 \mathrm{H}) ; 1.39\left(s, 18 \mathrm{H}, \mathrm{C}\left(\mathrm{CH}_{3}\right)_{3}\right) ; 1.09$ (s, $6 \mathrm{H}, \mathrm{CH}_{3}$ (TEMPO)); 1.02 (s, $6 \mathrm{H}, \mathrm{CH}_{3}$

(TEMPO)). ${ }^{13} \mathrm{C}-\mathrm{NMR}\left(75 \mathrm{MHz}, \mathrm{CDCl}_{3}\right)$ : both diastereoisomers: 168.6, 168.5, 168.4, 81.5, 77.9, 74.9, 73.1, 71.8, 71.3, 71.0, 59.8, 53.0, 52.8, 45.0, 40.9, 40.5, 39.65, 39.61, 39.1, 33.1, 32.0, 28.0, 27.9, 27.7, 26.6, 20.1, 17.0. MS (ESI): $492\left(95,[\mathrm{M}+\mathrm{Na}]^{+}\right), 470\left(100,[\mathrm{M}+\mathrm{H}]^{+}\right)$, HRMS (ESI): calcd for $\mathrm{C}_{26} \mathrm{H}_{48} \mathrm{NO}_{6}\left([\mathrm{M}+\mathrm{H}]^{+}\right)$: 469.3403. Found: 469.3403; corresponding telomer $29(\mathrm{n}=2)$ : MS (ESI): 568 (100, $\left[\mathrm{M}+\mathrm{H}^{+}\right]$), HRMS (ESI): calcd for $\mathrm{C}_{32} \mathrm{H}_{58} \mathrm{NO}_{7}$ ([M+H $\left.\left.\mathrm{H}^{+}\right]\right): 568.4213$. Found: 568.4228.

\section{2-[4-(2,2,6,6-Tetramethyl-piperidin-1-yloxymethyl)-1-(toluene-4-sulfonyl)-pyrrolidin-3-} ylmethyl]dimethylmalonate (30)

According to GP 3 with $10(68 \mathrm{mg}, 0.24 \mathrm{mmol})$ and $24(371 \mathrm{mg}, 0.71 \mathrm{mmol})$ in DCE (0.08 $\mathrm{mL})$ and purification by $\mathrm{FC}\left(\mathrm{SiO}_{2}\right.$, pentane/t-butylmethylether $\left.=2: 1\right)$ afforded $30(63 \mathrm{mg}$, 
49\%) as a 1.3:1 cis:trans mixture of diastereoisomers. I.R. (film): 3465w, 2930s, 1736s, $1598 w, 1437 m, 1346 m, 1163 s, 816 m, 665 m \mathrm{~cm} .{ }^{1} \mathrm{H}-\mathrm{NMR}(300 \mathrm{MHz})$ : both diastereoisomers: 7.72-7.69 (m, $2 \mathrm{H}$, aryl-H); 7.33-7.30 (m, $2 \mathrm{H}$, aryl-H); 3.72, $3.71\left(2 \mathrm{~s}, 6 \mathrm{H}, \mathrm{CH}_{3} \mathrm{O}\right)$; 3.83-3.59 ( $\left.m, 2 \mathrm{H}, \mathrm{CH}_{2} \mathrm{ON}\right) ; 3.51-2.88$ ( $\left.m, 8 \mathrm{H}, \mathrm{CH}_{2}, \mathrm{CH}\right) ; 2.43$ (s, $3 \mathrm{H}, \mathrm{PhCH}_{3}$ ); 1.80-1.00 (br $m, 18$ H,TEMPO). ${ }^{13} \mathrm{C}-\mathrm{NMR}$ (75 MHz): both diastereoisomers: 128.9, 126.8, 126.6, 73.7, 51.8, 50.2, 49.6, 39.4, 38.8, 37.5, 32.2, 28.9, 27.0, 26.5, 20.7, 19.2, 16.2. MS (ESI): 561 (100, $\left[\mathrm{M}+\mathrm{Na}^{+}\right), 539\left(25,[\mathrm{M}+\mathrm{H}]^{+}\right)$. HRMS (ESI) calcd for $\mathrm{C}_{27} \mathrm{H}_{42} \mathrm{~N}_{2} \mathrm{NaO}_{7} \mathrm{~S}\left(\left[\mathrm{M}+\mathrm{Na}^{+}\right): 561.2610\right.$. Found: 561.2608.

\section{2-[4-(2,2,6,6-Tetramethylpiperidin-1-yloxymethyl)-1-(toluene-4-sulfonyl)-pyrrolidin-3- ylmethyl]di-tert-butylmalonate (31)}

According to GP 3 with 11 (100 mg, $0.27 \mathrm{mmol})$ and 24 (406 mg, $0.81 \mathrm{mmol})$ in $t$ - $\mathrm{BuOH}$ and purification by $\mathrm{FC}\left(\mathrm{SiO}_{2}\right.$, pentane/t-butylmethylether $=4: 1, t$-butylmethylether $)$ afforded 31 (70 mg, 42\%) as a 1.5:1 cis:trans mixture of diastereoisomers and the corresponding telomer 31 (n=2) (55 mg, 23\%). I.R. (film): 3448w, 2976s, 2932s, 1727s, 1598m, 1472s, 1455s, $1393 s, 1347 s, 1256 s, 1094 s, 1043 s, 848 m, 816 s, 706 m, 664 s, 588 s, 549 s .{ }^{1} \mathrm{H}-\mathrm{NMR}(300$ $\mathrm{MHz}, \mathrm{CDCl}_{3}$ ): both diastereomeres: $7.63(\mathrm{~d}, J=12.4 \mathrm{~Hz}, 2 \mathrm{H}, \mathrm{H}$ (aromat)); 7.24 (d, J= 12.4 $\mathrm{Hz}, 2 \mathrm{H}, \mathrm{H}$ (aromat)); 3.75-2.80 (m, 7H); 2.36; 2.34 (2 s, $3 \mathrm{H}, \mathrm{CH}_{3}$ (tol)); 2.09-0.62 (m, $\left.10 \mathrm{H}\right)$; $1.38 ; 1.36 ; 1.353 ; 1.347\left(4 \mathrm{~s}, 18 \mathrm{H}, \mathrm{C}\left(\mathrm{CH}_{3}\right)_{3}\right) ; 0.99 ; 0.97 ; 0.94\left(3\right.$ br $s, 12 \mathrm{H}, 4 \mathrm{CH}_{3}$ (TEMPO)). ${ }^{13} \mathrm{C}-\mathrm{NMR}\left(75 \mathrm{MHz}, \mathrm{CDCl}_{3}\right)$ : both diastereomeres: 168.6, 168.3, 143.7, 143.5, 134.6, 134.0, 133.3, 129.9, 127.7, 127.4, 81.9, 77.7, 74.9, 60.0, 53.2, 52.8, 51.6, 50.9, 49.6, 44.1, 40.5, 39.3, 38.8, 34.3, 33.3, 31.8, 28.3, 28.1, 27.3, 27.2, 23.7, 21.7, 20.4, 17.3; MS (ESI): $623\left(100,[\mathrm{M}+\mathrm{H}]^{+}\right), 523$ (80). HRMS (ESI): calcd for $\mathrm{C}_{33} \mathrm{H}_{55} \mathrm{~N}_{2} \mathrm{O}_{7} \mathrm{~S}\left([\mathrm{M}+\mathrm{H}]^{+}\right)$: 623.3730. found: 623.3724; corresponding telomer $31(\mathrm{n}=2)$ : MS (ESI): $896\left(17,[\mathrm{M}+\mathrm{Na}]^{+}\right)$, $874\left(100,[\mathrm{M}+\mathrm{H}]^{+}\right)$. HRMS (ESI): calcd for $\mathrm{C}_{46} \mathrm{H}_{72} \mathrm{~N}_{3} \mathrm{O}_{9} \mathrm{~S}_{2}\left([\mathrm{M}+\mathrm{H}]^{+}\right)$: 874.4710. Found: 874.4727 .

\section{2-[8,8-Dimethyl-3-(2,2,6,6-tetramethyl-piperidin-1-yloxymethyl)-7,9-dioxa-spiro [4.5]} dec-2-ylmethyl]dimethylmalonate (32)

According to GP 3 with $10(77 \mathrm{mg}, 0.27 \mathrm{mmol})$ and $25(158 \mathrm{mg}, 0.81 \mathrm{mmol})$ in dichloroethane $(0.08 \mathrm{~mL})$ and purification by $\mathrm{FC}\left(\mathrm{SiO}_{2}\right.$, pentane/t-butylmethylether $\left.=4: 1\right)$ afforded 32 (45 mg, 36\%) as a 2.3:1 cis:trans mixture of diastereoisomers. I. R. (film): $2934 s$, $2866 s, 1737 s, 1437 s, 1372 s, 1261 s, 1199 s, 1154 s, 1080 m, 1030 m, 931 w, 831 m, 804 w, 733 m$, $520 w \mathrm{~cm}^{-1} .{ }^{1} \mathrm{H}-\mathrm{NMR}\left(200 \mathrm{MHz}, \mathrm{CDCl}_{3}\right)$ : both diastereomeres: 3.79 -3.50 (m, $\left.7 \mathrm{H}\right) ; 3.70$; 
$3.692 ; 3.687 ; 3.682\left(4 s, 6 \mathrm{H}, \mathrm{OCH}_{3}\right) ; 2.26-0.77(m, 14 \mathrm{H}) ; 1.40 ; 1.37 ; 1.36 ; 1.35(4 s, 6 \mathrm{H}$, $\mathrm{CH}_{3}$-C-O); $1.10 ; 1.05 ; 1.02$ (3 bs, $12 \mathrm{H}, \mathrm{CH}_{3}$ (TEMPO)). ${ }^{13} \mathrm{C}-\mathrm{NMR}\left(75 \mathrm{MHz}, \mathrm{CDCl}_{3}\right.$ ): both diastereomeres: 170.0, 169.7, 97.7, 97.6, 78.8, 77,4, 71.0, 70.0, 69.5, 69.2, 59.8, 59.7, 52.4, $52.3,50.8,50.7,44.5,40.9,40.5,40.4,40.2$, 39.72, 39.69, 38.6, 38.4, 37.0, 36.1, 34.1, 33.2, 33.1, 30.0, 25.3, 24.5, 23.1, 22.2, 20.2, 17.1. MS (ESI): 506 (74, [M+Na $\left.{ }^{+}\right], 484(100$, $\left.\left[\mathrm{M}+\mathrm{H}^{+}\right]\right)$. HRMS (ESI): calcd for $\mathrm{C}_{26} \mathrm{H}_{46} \mathrm{NO}_{7}\left(\left[\mathrm{M}+\mathrm{H}^{+}\right]\right)$: 484.327. Found: 484.329 .

\section{2-[8,8-Dimethyl-3-(2,2,6,6-tetramethyl-piperidin-1-yloxymethyl)-7,9-dioxa-spiro[4.5]dec-}

\section{2-ylmethyl]di-tert-butylmalonate (33)}

According to GP 3 with 11 (100 mg, $0.27 \mathrm{mmol})$ and 25 (158 mg, $0.81 \mathrm{mmol})$ in $t$-BuOH and purification by $\mathrm{FC}\left(\mathrm{SiO}_{2}\right.$, pentane/t-butylmethylether $=10: 1$, pentane/t-butylmethyl-ether $=$ $5: 1$, pentane/t-butylmethylether $=3: 1)$ afforded $33(52 \mathrm{mg}, 34 \%)$ as a 2.1:1 cis:trans mixture of diastereoisomers and the corresponding telomer $33(n=2)(24 \mathrm{mg}, 12 \%)$. I.R. (film): $2977 s, 2933 s, 2868 m, 1728 s, 1454 m, 1369 s, 1256 m, 1199 m, 1156 s, 1081 w, 1032 w, 919 w$, $848 w, 832 w, 734 m \mathrm{~cm}^{-1}$; ${ }^{1} \mathrm{H}-\mathrm{NMR}\left(200 \mathrm{MHz}, \mathrm{CDCl}_{3}\right)$ : both diastereomeres: $3.78-2.75(\mathrm{~m}, 7$ H); 2.24-0.79 ( $m, 14 \mathrm{H}) ; 1.43 ; 1.404 ; 1.402 ; 1.394 ; 1.387 ; 1.38 ; 1.36 ; 1.34\left(8 s, 24 \mathrm{H}, \mathrm{CH}_{3^{-}}\right.$ $\left.\mathrm{C}_{\mathrm{q}}-\mathrm{O}, \mathrm{C}\left(\mathrm{CH}_{3}\right)_{3}\right) ; 1.02 ; 1.03 ; 1.05$ (3 br $s, 12 \mathrm{H}, \mathrm{CH}_{3}$ (TEMPO)). ${ }^{13} \mathrm{C}-\mathrm{NMR}\left(75 \mathrm{MHz}, \mathrm{CDCl}_{3}\right)$ : both diastereomeres: 168.7, 168.6, 168.5, 97.25, 97.20, 80.8, 80.7, 79.5, 78.9, 78.8, 76.6, 76.2, 70.9, 70.8, 70.7, 69.8, 69.3, 69.2, 68.9, 59.4, 52.6, 52.5, 44.3, 43.7, 40.9, 40.6, 40.22, 40.16, 40.1, 40.0, 39.9, 39.6, 39.5, 38.3, 38.2, 37.2, 36.77, 35.9, 34.7, 34.5, 34.1, 33.2, 32.8, $32.7,29.7,29.3,29.0,27.7,27.6,27.42,27.38,25.8,24.9,24.5,24.4,22.6,22.4,22.0,20.0$, 16.7, 15.4. MS (ESI): 568 (100, $\left.\left[\mathrm{M}+\mathrm{H}^{+}\right]\right), 468$ (56). HRMS (ESI): calcd for $\mathrm{C}_{32} \mathrm{H}_{57} \mathrm{NO}_{7}$ $\left(\left[\mathrm{M}+\mathrm{H}^{+}\right]\right): 568.4213$. Found: 468.4233; corresponding telomer 33 ( $\left.\mathrm{n}=2\right)$ : MS (ESI): 786 (16, $\left.\left[\mathrm{M}+\mathrm{Na}^{+}\right]\right), 764\left(24,\left[\mathrm{M}+\mathrm{H}^{+}\right]\right)$. HRMS (ESI): calcd for $\mathrm{C}_{44} \mathrm{H}_{78} \mathrm{NO}_{9}\left([\mathrm{M}+\mathrm{H}]^{+}\right): 764.5677$. Found: 764.5656.

\section{2-(2-Methylennonyl)dimethylmalonate (34)}

According to GP 1, with 10 (100 mg, $0.348 \mathrm{mmol})$ and 2-methyl-1-nonene (244 mg, 1.74 $\mathrm{mmol}$ ) in DCE $(0.35 \mathrm{~mL})$. FC (ether/pentane = 1:20) afforded 34 (35 mg, 37\%). I.R. (film): $3468 w, 3319 w, 3267 w, 2928 m, 2856 m, 1739 s, 1647 w, 1436 m, 1222 s, 1149 s \mathrm{~cm}^{-1} .{ }^{1} \mathrm{H}-\mathrm{NMR}$ (400 MHz, $\left.\mathrm{CDCl}_{3}\right)$ : exo-methylene: $4.75\left(d, J=21.4 \mathrm{~Hz}, 2 \mathrm{H}, \mathrm{CH}_{2}\right) ; 3.72\left(s, 6 \mathrm{H}, \mathrm{CH}_{3}\right) ; 3.61$ $(t, J=7.9 \mathrm{~Hz}, 1 \mathrm{H}, \mathrm{CH}) ; 2.62\left(d, J=7.9 \mathrm{~Hz}, 2 \mathrm{H}, \mathrm{CH}_{2}\right) ; 2.00\left(t, J=7.3 \mathrm{~Hz}, 2 \mathrm{H}, \mathrm{CH}_{2}\right) ; 1.46-$ $1.38\left(m, 2 \mathrm{H}, \mathrm{CH}_{2}\right) ; 1.34-1.19\left(m, 8 \mathrm{H}, \mathrm{CH}_{2}\right) ; 0.87\left(t, J=6.3 \mathrm{~Hz}, 3 \mathrm{H}, \mathrm{CH}_{3}\right) ; \beta, \gamma$-unsaturated (mixture of E- and Z-isomer): $5.44(d, J=9.4 \mathrm{~Hz}, 1 \mathrm{H}, \mathrm{CH}) ; 4.29(d, J=9.6 \mathrm{~Hz}, 1 \mathrm{H}, \mathrm{CH}$, 
minor isomer); $4.27\left(d, J=9.4 \mathrm{~Hz}, 1 \mathrm{H}, \mathrm{CH}\right.$, major isomer); $3.71\left(s, 6 \mathrm{H}, \mathrm{CH}_{3}\right) ; 2.04(t, J=$ $\left.7.5 \mathrm{~Hz}, 2 \mathrm{H}, \mathrm{CH}_{2}\right)$; 1.61 ( $s, 3 \mathrm{H}, \mathrm{CH}_{3}$, minor isomer); 1.60 (s, $3 \mathrm{H}, \mathrm{CH}_{3}$, major isomer); 1.46$1.38\left(m, 2 \mathrm{H}, \mathrm{CH}_{2}\right) ; 1.34-1.19\left(m, 8 \mathrm{H}, \mathrm{CH}_{2}\right) ; 0.87\left(t, J=6.3 \mathrm{~Hz}, 3 \mathrm{H}, \mathrm{CH}_{3}\right) ; \gamma, \delta$-unsaturated (mixture of E- and Z-isomer): $5.19(t, J=7.9 \mathrm{~Hz}, 1 \mathrm{H}, \mathrm{CH}) ; 3.70\left(s, 6 \mathrm{H}, \mathrm{CH}_{3}\right) ; 3.54(t, J=$ $5.4 \mathrm{~Hz}, 1 \mathrm{H}, \mathrm{CH}$, major isomer); $3.53(t, J=7.9 \mathrm{~Hz}, 1 \mathrm{H}, \mathrm{CH}$, minor isomer); 2.65 ( $d, J=7.9$ $\mathrm{Hz}, 1 \mathrm{H}, \mathrm{CH}_{2}$, minor isomer); $2.57\left(d, J=7.9 \mathrm{~Hz}, 1 \mathrm{H}, \mathrm{CH}_{2}\right.$, major isomer); 1.93 ( $q, J=6.8$ $\left.\mathrm{Hz}, 2 \mathrm{H}, \mathrm{CH}_{2}\right) ; 1.76$ ( $s, 3 \mathrm{H}, \mathrm{CH}_{3}$, minor isomer); 1.67 ( $s, 3 \mathrm{H}, \mathrm{CH}_{3}$, major isomer); 1.46-1.38 $\left(m, 2 \mathrm{H}, \mathrm{CH}_{2}\right) ; 1.34-1.19\left(m, 6 \mathrm{H}, \mathrm{CH}_{2}\right) ; 0.87\left(t, J=6.3 \mathrm{~Hz}, 3 \mathrm{H}, \mathrm{CH}_{3}\right) \cdot{ }^{13} \mathrm{C}-\mathrm{NMR}(100 \mathrm{MHz}$, $\left.\mathrm{CDCl}_{3}\right)$ : exo-methylene: $169.5(\mathrm{C}), 145.8(\mathrm{C}), 110.9\left(\mathrm{CH}_{2}\right), 52.6\left(\mathrm{CH}_{3}\right), 52.4\left(\mathrm{CH}_{3}\right), 50.4$ $(\mathrm{CH}), 35.9\left(\mathrm{CH}_{2}\right), 34.8\left(\mathrm{CH}_{2}\right), 31.8\left(\mathrm{CH}_{2}\right), 29.2\left(\mathrm{CH}_{2}\right), 29.1\left(\mathrm{CH}_{2}\right), 27.6\left(\mathrm{CH}_{2}\right), 22.6\left(\mathrm{CH}_{2}\right)$, 14.1 $\left(\mathrm{CH}_{3}\right)$. MS (ESI): $309\left(14,[\mathrm{M}+\mathrm{K}]^{+}\right), 293\left(100,[\mathrm{M}+\mathrm{Na}]^{+}\right), 271\left(8,[\mathrm{M}+\mathrm{H}]^{+}\right), 207(14)$, 158 (15), 126 (32). HRMS (ESI) calcd for $\mathrm{C}_{15} \mathrm{H}_{26} \mathrm{O}_{4} \mathrm{Na}\left([\mathrm{M}+\mathrm{Na}]^{+}\right)$: 293.1729. Found: 293.1722 .

\section{2-(2-Methylennonyl)di-tert-butylmalonate (35)}

According to GP 2, with 11 (100 mg, $0.27 \mathrm{mmol})$ and 2-methyl-1-nonene (189 mg, 1.35 $\mathrm{mmol})$ in $t$ - $\mathrm{BuOH}(0.27 \mathrm{~mL}) . \mathrm{FC}$ (ether/pentane $=1: 50)$ afforded $35(53 \mathrm{mg}, 56 \%)$. I.R. (film): 3442m, 3931s, 2873m, 1731s, 1458m, 1369m, 1256w, 1140m, $\mathrm{cm}^{-1}$. ${ }^{1} \mathrm{H}-\mathrm{NMR}$ (400 $\left.\mathrm{MHz}, \mathrm{CDCl}_{3}\right)$ : exo-methylene: $4.75\left(d, J=9.0 \mathrm{~Hz}, 2 \mathrm{H}, \mathrm{CH}_{2}\right) ; 3.36(t, J=7.7 \mathrm{~Hz}, 1 \mathrm{H}, \mathrm{CH})$; $2.51\left(d, J=7.7 \mathrm{~Hz}, 2 \mathrm{H}, \mathrm{CH}_{2}\right) ; 2.01\left(t, J=8.0 \mathrm{~Hz}, 2 \mathrm{H}, \mathrm{CH}_{2}\right) ; 1.51-1.37\left(m, 2 \mathrm{H}, \mathrm{CH}_{2}\right) ; 1.44$ $\left(s, 18 \mathrm{H}, \mathrm{CH}_{3}\right) ; 1.33-1.21\left(m, 8 \mathrm{H}, \mathrm{CH}_{2}\right) ; 0.88-0.85\left(m, 3 \mathrm{H}, \mathrm{CH}_{3}\right) ; \beta$, $\gamma$-unsaturated (mixture of E- and Z-isomer): $5.41\left(d \times d, J_{1}=9.2 \mathrm{~Hz}, J_{2}=1.3 \mathrm{~Hz}, 1 \mathrm{H}, \mathrm{CH}\right) ; 4.04(d, J=9.6 \mathrm{~Hz}, 1 \mathrm{H}$, $\mathrm{CH}$, minor isomer); $4.02(d, J=9.2 \mathrm{~Hz}, 1 \mathrm{H}, \mathrm{CH}$, major isomer); $2.04(t, J=6.8 \mathrm{~Hz}, 2 \mathrm{H}$, $\left.\mathrm{CH}_{2}\right) ; 1.65\left(d, J=1.0 \mathrm{~Hz}, 3 \mathrm{H}, \mathrm{CH}_{3}\right.$, major isomer $) ; 1.60\left(d, J=0.6 \mathrm{~Hz}, 3 \mathrm{H}, \mathrm{CH}_{3}\right.$, minor isomer); $1.51-1.37\left(m, 2 \mathrm{H}, \mathrm{CH}_{2}\right) ; 1.45\left(s, 18 \mathrm{H}, \mathrm{CH}_{3}\right) ; 1.33-1.21\left(m, 8 \mathrm{H}, \mathrm{CH}_{2}\right) ; 0.88-0.85$ ( $\left.m, 3 \mathrm{H}, \mathrm{CH}_{3}\right) ; \gamma, \delta$-unsaturated (mixture of E- and Z-isomer): $5.19\left(d \times d, J_{1}=6.4 \mathrm{~Hz}, J_{2}=5.8\right.$ $\mathrm{Hz}, 1 \mathrm{H}, \mathrm{CH}) ; 3.33(t, J=7.7 \mathrm{~Hz}, 1 \mathrm{H}, \mathrm{CH}$, major isomer $) ; 3.31(t, J=7.7 \mathrm{~Hz}, 1 \mathrm{H}, \mathrm{CH}$, minor isomer); $2.55\left(d, J=7.9 \mathrm{~Hz}, 2 \mathrm{H}, \mathrm{CH}_{2}\right.$, minor isomer); $2.47\left(d, J=7.9 \mathrm{~Hz}, 2 \mathrm{H}, \mathrm{CH}_{2}\right.$, major isomer); $1.94\left(q, J=6.4 \mathrm{~Hz}, 2 \mathrm{H}, \mathrm{CH}_{2}\right) ; 1.75\left(d, J=1.3 \mathrm{~Hz}, 3 \mathrm{H}, \mathrm{CH}_{3}\right.$, major isomer); 1.68 (d, $J=0.8 \mathrm{~Hz}, 3 \mathrm{H}, \mathrm{CH}_{3}$, minor isomer); $1.51-1.37\left(m, 2 \mathrm{H}, \mathrm{CH}_{2}\right) ; 1.43\left(s, 18 \mathrm{H}, \mathrm{CH}_{3}\right) ; 1.33-1.21$ $\left(m, 6 \mathrm{H}, \mathrm{CH}_{2}\right) ; 0.88-0.85\left(m, 6 \mathrm{H}, \mathrm{CH}_{3}\right) .{ }^{13} \mathrm{C}-\mathrm{NMR}\left(100 \mathrm{MHz}, \mathrm{CDCl}_{3}\right)$ : exo-methylene: 168.6 (C), $168.5(\mathrm{C}), 146.2(\mathrm{C}), 110.6\left(\mathrm{CH}_{2}\right), 81.3(\mathrm{C}), 81.1(\mathrm{C}), 52.4(\mathrm{CH}), 35.9\left(\mathrm{CH}_{2}\right), 34.7\left(\mathrm{CH}_{2}\right)$, $31.8\left(\mathrm{CH}_{2}\right), 29.6\left(\mathrm{CH}_{2}\right), 29.2\left(\mathrm{CH}_{2}\right), 27.9\left(\mathrm{CH}_{3}\right), 27.6\left(\mathrm{CH}_{2}\right), 22.6\left(\mathrm{CH}_{2}\right), 14.1\left(\mathrm{CH}_{3}\right) . \mathrm{MS}$ (ESI): $393\left(16,[\mathrm{M}+\mathrm{K}]^{+}\right), 377\left(100,[\mathrm{M}+\mathrm{Na}]^{+}\right), 321$ (43), 265 (28), 243 (45), 126 (22). HRMS 
(ESI) calcd for $\mathrm{C}_{21} \mathrm{H}_{38} \mathrm{NaO}_{4}\left([\mathrm{M}+\mathrm{Na}]^{+}\right)$: 377.2668. Found: 377.2663.

\section{3-(2,2-Bis-methoxycarbonyl-ethyl)-4-isopropenyl-cyclopentane-1,1-dicarboxylic acid} dimethyl ester (37)

A solution of $10(100 \mathrm{mg}, 0.35 \mathrm{mmol})$ and $36(504 \mathrm{mg}, 2.10 \mathrm{mmol})$ in DCE $(0.16 \mathrm{~mL})$ was heated for $72 \mathrm{~h}$ at $135^{\circ} \mathrm{C}$ (sealed tube). Removal of the solvent and purification by $\mathrm{FC}\left(\mathrm{SiO}_{2}\right.$, pentane/t-butylmethylether $=4: 1)$ provided $37(55 \mathrm{mg}, 42 \%)$ as a 2.4:1 cis:trans mixture of diastereoisomers. I.R. (film): 3470w, 2955s, 1735s, 1647w, 1436s, 1375w, 1257s, 1198s, $1153 s, 1058 m, 915 w, 734 w .{ }^{l} \mathrm{H}-\mathrm{NMR}\left(200 \mathrm{MHz}, \mathrm{CDCl}_{3}\right)$ : both diastereomeres: $3.85-3.36$ ( $m$, $2 \mathrm{H}) ; 3.72 ; 3.70 ; 3.694 ; 3.688 ; 3.67\left(5 s, 12 \mathrm{H}, \mathrm{OCH}_{3}\right), 3.43-3.36$ (m, $\left.1 \mathrm{H}, \mathrm{C} \underline{\mathrm{H}}-\mathrm{COOMe}\right)$; 2.61-0.83 (m, $6 \mathrm{H}) ; 1.70\left(s, 3 \mathrm{H}, \mathrm{CH}_{3}-\mathrm{C}_{\mathrm{sp} 2}\right) .{ }^{13} \mathrm{C}-\mathrm{NMR}\left(75 \mathrm{MHz}, \mathrm{CDCl}_{3}\right)$ : both diastereomeres: 172.9, 172.7, 172.3, 169.8, 169.6, 169.5, 144.0, 143.4, 112.8, 111.7, 58.3, 58.0, 55.1, 53.5, 52.8, 52.7, 52.5, 52.4, 52.2, 51.3, 50.4, 49.8, 49.3, 44.6, 44.3, 39.9, 39.6, 39.4, 39.3, 37.85, 37.77, 35.6, 35.4, 33.2, 33.0, 32.6, 31.0, 29.2, 28.3, 26.1, 22.9, 20.2, 20.1, 18.6, 17.0, 15.1. MS (EI): 371 (34 [M+H']), 339 (70), 307 (86), 278 (85), 238 (100), 178 (85). HRMS (EI): calcd for $\mathrm{C}_{18} \mathrm{H}_{26} \mathrm{O}_{8}\left(\left[\mathrm{M}+\mathrm{H}^{+}\right]\right): 371.1706$. found: 371.1696 .

\section{2-(4-Isopropenylcyclohex-1-enylmethyl)dimethylmalonate (38)}

According to GP 1, with 10 (100 mg, $0.348 \mathrm{mmol})$ and (1S)-(-)-( $\beta$ )-pinene (237 mg, 1.74 mmol) in DCE (0.35 mL). FC (ether/pentane $=1: 20$ to $1: 10)$ afforded 38 (51 mg, 55\%). I.R. (film): $3462 w, 3081 w, 2953 s, 2921 s, 2840 m, 1739 s, 1644 m, 1436 s, 1346 m, 1234 s, 1153 m \mathrm{~cm}^{-}$ 1. ${ }^{1} \mathrm{H}-\mathrm{NMR}\left(200 \mathrm{MHz}, \mathrm{CDCl}_{3}\right): 5.46(s b r, 1 \mathrm{H}, \mathrm{CH}) ; 4.69-4.67\left(m, 2 \mathrm{H}, \mathrm{CH}_{2}\right) ; 3.72(s, 6 \mathrm{H}$, $\left.\mathrm{CH}_{3}\right) ; 3.57(t, J=7.8 \mathrm{~Hz}, 1 \mathrm{H}, \mathrm{CH}) ; 2.55\left(d, J=7.8 \mathrm{~Hz}, 2 \mathrm{H}, \mathrm{CH}_{2}\right) ; 2.11-1.77(m, 5 \mathrm{H}) ; 1.71$ $\left(s, 3 \mathrm{H}, \mathrm{CH}_{3}\right) ; 1.53-1.07(m, 2 \mathrm{H}) .{ }^{13} \mathrm{C}-\mathrm{NMR}\left(50 \mathrm{MHz}, \mathrm{CDCl}_{3}\right): 169.6$ (2x C), $149.7(\mathrm{C})$, $133.3(\mathrm{C}), 123.3(\mathrm{CH}), 108.6\left(\mathrm{CH}_{2}\right), 52.4(\mathrm{CH}), 50.4(2 \mathrm{x} \mathrm{CH}), 40.7(\mathrm{CH}), 36.5\left(\mathrm{CH}_{2}\right), 30.6$ $\left(\mathrm{CH}_{2}\right), 28.3\left(\mathrm{CH}_{2}\right), 27.6\left(\mathrm{CH}_{2}\right), 20.7\left(\mathrm{CH}_{3}\right)$. MS (ESI): 289 (100, [M+Na] $\left.]^{+}\right), 235$ (9), 135 (27). HRMS (ESI) calcd for $\mathrm{C}_{15} \mathrm{H}_{22} \mathrm{NaO}_{4}\left([\mathrm{M}+\mathrm{Na}]^{+}\right)$: 289.1416. Found: 289.1404.

\section{2-(4-Isopropenylcyclohex-1-enylmethyl)di-tert-butylmalonate (39)}

According to GP 2, with 11 (50 mg, $0.13 \mathrm{mmol}$ ) and (1S)-(-)-( $\beta$ )-pinene (91 mg, $0.67 \mathrm{mmol}$ ) in $t$ - $\mathrm{BuOH}(0.13 \mathrm{~mL})$. FC (ether/pentane = 1:50) afforded 39 (34 mg, 75\%). I.R. (film): $3476 w, 2978 s, 2931 s, 2838 m, 1730 s, 1644 w, 1456 m, 1249 s, 1136 s \mathrm{~cm}^{-1} .{ }^{1} \mathrm{H}-\mathrm{NMR}(300 \mathrm{MHz}$, $\left.\mathrm{CDCl}_{3}\right)$ : $5.46(m, 1 \mathrm{H}, \mathrm{CH}) ; 4.69-4.67\left(m, 2 \mathrm{H}, \mathrm{CH}_{2}\right) ; 3.33(t, J=7.3 \mathrm{~Hz}, 1 \mathrm{H}, \mathrm{CH}) ; 2.44(d, J$ $\left.=7.3 \mathrm{~Hz}, 2 \mathrm{H}, \mathrm{CH}_{2}\right) ; 2.14-1.62(m, 5 \mathrm{H}) ; 1.71\left(s, 3 \mathrm{H}, \mathrm{CH}_{3}\right) ; 1.43\left(s, 18 \mathrm{H}, \mathrm{CH}_{3}\right) ; 1.25-0.78$ 
(m, $2 \mathrm{H}) .{ }^{13} \mathrm{C}-\mathrm{NMR}\left(75 \mathrm{MHz}, \mathrm{CDCl}_{3}\right)$ : 168.7 (2x C), 149.9 (C), $133.8(\mathrm{C}), 123.0(\mathrm{CH}), 108.5$ $\left(\mathrm{CH}_{2}\right), 81.2(3 \mathrm{x} \mathrm{CH}), 52.4(\mathrm{CH}), 41.0(\mathrm{CH}), 36.5\left(\mathrm{CH}_{2}\right), 30.7\left(\mathrm{CH}_{2}\right), 28.4\left(\mathrm{CH}_{2}\right), 27.9\left(\mathrm{CH}_{2}\right)$, $20.8\left(\mathrm{CH}_{3}\right)$. MS (ESI): 373 (100, [M+Na] $\left.]^{+}\right), 317$ (22), 261 (19), 158 (37). HRMS (ESI) calcd for $\mathrm{C}_{21} \mathrm{H}_{34} \mathrm{NaO}_{4}\left([\mathrm{M}+\mathrm{Na}]^{+}\right)$: 373.2355. Found: 373.2338 .

\section{2-Hexyl-2-oxotetrahydrofuran-3-carboxylic acid methylester (40)}

To the alkoxyamine $12(150 \mathrm{mg}, 0.375 \mathrm{mmol})$ dissolved in THF (1.2 mL) was added activated $\mathrm{Zn}$ dust $(294 \mathrm{mg}, 4.50 \mathrm{mmol})$, water $(1.2 \mathrm{~mL})$ and conc. acetic acid $(3.7 \mathrm{~mL})$. The mixture was heated to $85{ }^{\circ} \mathrm{C}$ for $6 \mathrm{~h}$. After cooling to $0{ }^{\circ} \mathrm{C}$, the reaction mixture was treated with $1 \mathrm{~N} \mathrm{NaOH}$, then extracted with ether. Drying over $\mathrm{MgSO}_{4}$ and removal of the solvents provided the crude product which was purified by $\mathrm{FC}\left(\mathrm{SiO}_{2}\right.$, ether/pentane $\left.=1: 3\right)$ to afford the product 40 (62 mg, 73\%, dr = 1:1) as a colorless oil. I.R. (film): 3468w, 2932s, 2859m, 1780s, $1742 s, 1456 w, 1437 w, 1355 m, 1263 m, 1165 m \mathrm{~cm}^{-1} .{ }^{1} \mathrm{H}-\mathrm{NMR}$ (400 MHz, $\mathrm{CDCl}_{3}$ ): Isomer A: $4.63\left(d \times q, J_{1}=7.2 \mathrm{~Hz}, J_{2}=5.6 \mathrm{~Hz}, 1 \mathrm{H}, \mathrm{CH}\right) ; 3.76\left(s, 3 \mathrm{H}, \mathrm{CH}_{3}\right) ; 3.58\left(d \times d, J_{1}=9.6 \mathrm{~Hz}, J_{2}=\right.$ $4.9 \mathrm{~Hz}, 1 \mathrm{H}, \mathrm{CH}) ; 2.70-2.64\left(m, 1 \mathrm{H}, \mathrm{CH}_{2}\right) ; 2.11-2.03\left(m, 1 \mathrm{H}, \mathrm{CH}_{2}\right) ; 1.83-1.53(m, 2 \mathrm{H}$, $\left.\mathrm{CH}_{2}\right) ; 1.48-1.19\left(m, 8 \mathrm{H}, \mathrm{CH}_{2}\right) ; 0.85\left(t, J=6.5 \mathrm{~Hz}, 3 \mathrm{H}, \mathrm{CH}_{3}\right)$. Isomer B: 4.45-4.38 (m, $1 \mathrm{H}$, $\mathrm{CH}) ; 3.78\left(s, 3 \mathrm{H}, \mathrm{CH}_{3}\right) ; 3.61\left(d \times d, J_{l}=11.4 \mathrm{~Hz}, J_{2}=9.4 \mathrm{~Hz}, 1 \mathrm{H}, \mathrm{CH}\right) ; 2.56-2.49(m, 1 \mathrm{H}$, $\left.\mathrm{CH}_{2}\right) ; 2.33-2.24\left(m, 1 \mathrm{H}, \mathrm{CH}_{2}\right) ; 1.83-1.53\left(m, 2 \mathrm{H}, \mathrm{CH}_{2}\right) ; 1.48-1.19\left(m, 8 \mathrm{H}, \mathrm{CH}_{2}\right) ; 0.85(t, J$ $\left.=6.5 \mathrm{~Hz}, 3 \mathrm{H}, \mathrm{CH}_{3}\right) \cdot{ }^{13} \mathrm{C}-\mathrm{NMR}\left(50 \mathrm{MHz}, \mathrm{CDCl}_{3}\right)$ : Isomer A: $171.6(\mathrm{C}), 168.2(\mathrm{C}), 80.3(\mathrm{CH})$, $53.0\left(\mathrm{CH}_{3}\right), 46.7\left(\mathrm{CH}_{2}\right), 35.4\left(\mathrm{CH}_{2}\right), 31.9\left(\mathrm{CH}_{2}\right), 31.5\left(\mathrm{CH}_{2}\right), 28.8\left(\mathrm{CH}_{2}\right), 25.1\left(\mathrm{CH}_{2}\right), 22.4$ $\left(\mathrm{CH}_{2}\right), 13.9\left(\mathrm{CH}_{3}\right)$. Isomer B: $171.7(\mathrm{C}), 168.3(\mathrm{C}), 79.6(\mathrm{CH}), 52.0\left(\mathrm{CH}_{3}\right), 47.2\left(\mathrm{CH}_{2}\right), 35.2$ $\left(\mathrm{CH}_{2}\right), 32.1\left(\mathrm{CH}_{2}\right), 31.5\left(\mathrm{CH}_{2}\right), 28.8\left(\mathrm{CH}_{2}\right), 25.1\left(\mathrm{CH}_{2}\right), 22.4\left(\mathrm{CH}_{2}\right), 13.9\left(\mathrm{CH}_{3}\right)$. MS (EI): 228 (5.5, [M] $\left.]^{+}\right), 159$ (19), 143 (100), 111 (94), 87 (54). HRMS (EI) calcd for $\mathrm{C}_{12} \mathrm{H}_{20} \mathrm{O}_{4}\left([\mathrm{M}]^{+}\right)$: 228.1362. Found: 228.1359. 